\title{
Microstructure selection in thin-sample directional solidification of an Al-Cu alloy: In situ X-ray imaging and phase-field simulations
}

\author{
A.J. Clarke ${ }^{1,2 *}$, D. Tourret ${ }^{1 *}$, Y. Song ${ }^{3}$, S.D. Imhoff ${ }^{1}$, P.J. Gibbs ${ }^{1}$, J.W. Gibbs ${ }^{1}$, K. Fezzaa ${ }^{4}$, A. Karma ${ }^{3}$ \\ ${ }^{1}$ Los Alamos National Laboratory, Sigma Division, Los Alamos, NM, USA \\ ${ }^{2}$ George S. Ansell Department of Metallurgical and Materials Engineering, \\ Colorado School of Mines, Golden, CO, USA \\ ${ }^{3}$ Department of Physics and Center for Interdisciplinary Research on Complex Systems, \\ Northeastern University, Boston, MA, USA \\ ${ }^{4}$ Advanced Photon Source, Argonne National Laboratory, Lemont, IL, USA
}

\begin{abstract}
We study microstructure selection during during directional solidification of a thin metallic sample. We combine in situ X-ray radiography of a dilute $\mathrm{Al}-\mathrm{Cu}$ alloy solidification experiments with three-dimensional phase-field simulations. We explore a range of temperature gradient $G$ and growth velocity $V$ and build a microstructure selection map for this alloy. We investigate the selection of the primary dendritic spacing $\Lambda$ and tip radius $\rho$. While $\rho$ shows a good agreement between experimental measurements and dendrite growth theory, with $\rho \sim V^{-1 / 2}, \Lambda$ is observed to increase with $V(\partial \Lambda / \partial V>0)$, in apparent disagreement with classical scaling laws for primary dendritic spacing, which predict that $\partial \Lambda / \partial V<0$. We show through simulations that this trend inversion for $\Lambda(V)$ is due to liquid convection in our experiments, despite the thin sample configuration. We use a classical diffusion boundary-layer approximation to semi-quantitatively incorporate the effect of liquid convection into phase-field simulations. This approximation is implemented by assuming complete solute mixing outside a purely diffusive zone of constant thickness that surrounds the solid-liquid interface. This simple method enables us to quantitatively match experimental measurements of the planar morphological instability threshold and primary spacings over an order of magnitude in $V$. We explain the observed inversion of $\partial \Lambda / \partial V$ by a combination of slow transient dynamics of microstructural homogenization and the influence of the sample thickness.
\end{abstract}

Keywords: Directional solidification, Microstructure formation, X-ray radiography, Phase-field method.

\section{Introduction}

Microstructural characteristics, such as dendritic spacing, determine the properties, performance, and lifetime of metal cast parts. For most industrially relevant metallic alloys, solidification is the first processing

${ }^{*}$ Corresponding authors; Email: amyclarke@mines.edu (AJC), dtourret@lanl.gov (DT) 
step accompanied by microstructure formation. Solidification is also a prime example of nonlinear pattern formation $[1,2]$, where complexity stems from the broad range of length and time scales involved [3, 4]. Even though our understanding of crystal growth and solidification as a whole has evolved significantly over the past decades $[5,6]$, microstructure selection mechanisms are still not completely understood.

Many experimental studies of solidification patterns have made use of transparent organic compounds that solidify in a similar fashion as metallic alloys [7]. Such in situ solidification observations have led to significant insights into dendrite growth in undercooled melts $[8,9]$ as well as the formation of cellular and dendritic arrays during directional solidification of binary alloys [10,11, 12, 13, 14, 15, 16, 17, 18].

Synchrotron X-ray facilities around the world now provide intense and coherent X-rays, opening the door to similar in situ observations of metals and metallic alloys during solidification, with ever increasing spatial and temporal resolutions [19, 20,21]. Aluminum alloys have proven to be particularly good candidates for in situ X-ray observations and have been extensively used in metal solidification experiments (e.g. [19, 21, $22,23,24,25,26])$.

Both in experiments and simulations, the concept of microstructure selection maps as a function of temperature gradient $G$ and growth velocity $V$, as illustrated schematically in Fig. 1 (see e.g. Ref. [27]), has existed for decades [28]. In a diffusive regime, the critical velocity $V_{c}$ for the onset of planar morphological instability [29] may be estimated by the constitutional undercooling criterion [30] (see Sec. 4.2). The transition from cellular to dendritic growth occurs over a diffuse range around a transition velocity $V_{C D}$ that may be estimated by the limit of stability of cells as $V_{c}(1+k) / k$ [31], i.e. close to $V_{c} / k$ when the solute partition coefficient at the interface $k$ is small [13].

In terms of dendritic tip shape, it is now commonly accepted that for given growth conditions, e.g. a given solute concentration $c_{\infty}$, a given temperature gradient $G$, and a given growth velocity $V$, the selection of dendritic tip radius is unique, and follows microscopic solvability theory $[1,32,33]$. In contrast, cellular growth leads to a cell tip radius essentially proportional to the primary cellular spacing (see e.g. [34]).

In contrast to dendrite tip radius selection that occurs over a narrow distribution range, similar conditions can lead to primary cellular or dendritic spacings within a wide range, which has been shown both experimentally and theoretically $[11,12,13,14,15,16,17,27,35,36]$. However, a similar processing history usually results in similar spacings $[17,37]$. Microstructures may also exhibit oscillatory states close to spacing stability limits during both cellular [38, 39, 40, 41] and eutectic growth [39, 42, 43].

The mechanisms associated with the lower and upper spacing instability are well identified (see e.g. $[11,18,27,35,44,45])$, namely cell/dendrite elimination below the lower limit $\Lambda_{\min }$, and cell tip-splitting or dendritic tertiary branching above the upper limit $\Lambda_{\max }$. In early modeling studies, Hunt and Lu suggested that the upper spacing stability limit $\Lambda_{\max }$ had to be at least twice the lower limit $\Lambda_{\min }$, so that the spacing resulting from tip splitting or branching (hence half of the initial spacing) would remain higher than $\Lambda_{\min }$ [35]. However, they pointed out that it was unclear what could be used at the time to model 
the maximum dendrite spacing, and also suggested that in practice the average spacing was likely to be closer to the lower spacing limit than the upper spacing limit [35]. Since dendritic branching could not be treated with their model, they assumed the maximum dendrite spacing limit to be twice the lower stability limit [27]. This assumption has led to good agreement with experimentally measured distributions [27, 35]. It has since then been commonly assumed that $\Lambda_{\max } / \Lambda_{\min } \approx 2$ (see e.g. $[44,46]$ ) and later theoretical studies have focused mostly on the lower limit of spacing stability. Yet, recent simulations using quantitative phasefield $[34,45]$ and multiscale dendritic needle network modeling $[47,48]$ have shown that the upper spacing stability limit $\Lambda_{\max }$ may actually be a factor of 3 to 4 larger than $\Lambda_{\min }$ in a dendritic regime. However, most of the dynamically selected spacings occupy the lower half of the stability range, hence remaining between $\Lambda_{\min }$ and about $2 \Lambda_{\min }$.

In the immediate vicinity of the critical velocity $V_{c}$ corresponding to the onset of morphological instability, the average selected primary spacing $\Lambda(V)$ typically shows a sharp decrease with increasing $V$, followed by an increase of $\Lambda$ with $V$ in the cellular regime, up to the cell-to-dendrite transition, which occurs over a finite range of velocity around $V \approx V_{C D}$, and finally a decrease of $\Lambda$ with $V$ in the dendritic regime [49, 50] (for such observations in $\mathrm{Al}-\mathrm{Cu}$ alloys, see $[51,52]$ ). Thus, the $\Lambda(V)$ curve typically exhibits a maximum in the vicinity of the cell-to-dendrite transition at $V \approx V_{C D}$. In the dendritic regime the average primary spacing then decreases as $\Lambda \sim V^{\alpha}$, typically with $\alpha \approx-0.25[13,53]$.

Additionally, the aforementioned studies have focused mostly on purely diffusive transport conditions. Yet, significant convection may take place in liquid alloys, due to both thermal and solutal gradients, combined with the effect of gravity $[16,51,54,55,56,57,58]$. Convection has been shown to play an important role in microstructure selection in aluminum-copper (Al-Cu) alloys [51, 52, 59, 60, 61], even in thin sample experiments in which confinement is intended to limit the extent of convection [62, 63, 64].

In terms of microstructure selection, liquid convective transport is understood to strongly affect heat and/or mass transport but to have a negligible influence on the dendrite tip operating state $[65,66,67,68$, $69,70,71]$. On the other hand, gravity-induced liquid convection is known to have a significant impact on dendritic spacing selection. However, most theoretical studies of directional solidification with convection to date have been performed on relatively limited sample sizes and in two dimensions (2D) [61, 72].

In the current study, we combine synchrotron X-ray in situ observations of a dilute $\mathrm{Al}$-Cu alloy solidification with quantitative phase-field $(\mathrm{PF})$ simulations in 3D to explore microstructure selection mechanisms in thin sample directional solidification. We explore a range of temperature gradients and growth velocities to build a morphological selection map analogous to Fig. 1 and systematically measure cellular and dendritic tip radii and primary spacings. We compare these measurements quantitatively with existing analytical theories and PF simulations that incorporate approximately the effect of convection using a diffusion boundary-layer approximation. Despite its simplicity, this approximation has the advantage that it allows us to simulate the formation of spatially extended cellular/dendritic arrays on experimentally relevant length and time scales. 
Furthermore, it reproduces well salient features of the experiments including a strong effect of convection on the limit of planar stability and on the spacing-velocity relationship.

\section{Experiments}

\subsection{Sample preparation}

We prepared an $\mathrm{Al}-0.6$ at.\% $\mathrm{Cu}$ alloy (i.e. 1.4wt\% Cu), using high purity (99.999\%) Al and Cu by arcmelting. The buttons were flipped and remelted at least five times to ensure homogeneity. Thin samples were rolled to a thickness $(z)$ of about $200 \mu \mathrm{m}$. Individual samples with $(x \times y)$ dimensions of about $30 \times 10 \mathrm{~mm}^{2}$ were sheared for X-ray imaging experiments.

\subsection{Directional solidification}

Samples were directionally solidified at a constant velocity within an imposed temperature gradient with a standard power down method, directly controlling the temperature gradient and the cooling rate.

Each sample was placed in a boron nitride crucible with a $2 \mathrm{~mm}$ diameter observation window that is thinned to $0.1 \mathrm{~mm}$ thickness (see additional pictures and schematics in the Supplementary Material of Ref. [26]). The crucible containing the sample was then inserted into a slotted steel rod with a hole along the X-ray beam path. The steel rod was heated by two independently controlled induction coils above and below the sample position. The rod was equipped with 12 thermocouples at different locations to provide measurements of the thermal conditions. The rod was heated and the temperature gradient was stabilized before reducing the power in the coils. Feedback control from the thermocouples was used to ensure and maintain a constant cooling rate and temperature gradient across the sample. Unless mentioned otherwise (i.e. only in Sec. 4.1), experiments were done with an upward growth direction, i.e. opposite to gravity.

We explored different temperature gradients, from $G \approx 50$ to $85 \mathrm{~K} / \mathrm{cm}$ and a range of growth velocities from $V \approx 1$ to $65 \mu \mathrm{m} / \mathrm{s}$. For this alloy, the range of $G$ and $V$ is expected to fall within the domain schematically represented by the gray rectangle in Fig. 1. The current study constitutes a total of over 50 directional solidification experiments, thus compiling slightly more than 450 measured primary dendrite tip radii and primary spacings (measured as described in Sec. 2.4).

\subsection{In situ $X$-ray synchrotron imaging}

In order to perform in situ synchrotron X-ray imaging during the experiments, the directional solidification setup was installed at the Sector 32 Insertion Device beam line at Argonne National Laboratory's Advanced Photon Source. During the experiments, a monochromatic beam of $18 \mathrm{keV}$ X-rays passed through metallic alloy samples, impinged upon a scintillator that converts X-rays to visible light, captured by a CCD camera recording $1024 \times 1280$ pixel 12 bit images with a pixel size of $1.34 \mu \mathrm{m}$ at a frame rate between 3 to 
$5 \mathrm{~Hz}$, depending on the experiment. Due to the small difference in composition between the liquid and solid phases in this dilute alloy, the contrast from X-ray absorption is minimal. Instead, we use phase contrast, which is generated by using a coherent X-ray source and separating the sample and the scintillator by about a meter.

\subsection{Image post-processing}

For each experiment, we extracted the solidification front growth velocity from time-resolved image processing and the temperature gradient from the thermocouple measurements.

Images acquired from the experiments were first subjected to standard processing routines (i.e. alignment and background subtraction). Because the contrast in the images is due to phase contrast, there is relatively little difference in the gray levels between the liquid and the solid, but there are bright bands where the interface is tangential to the beam path. To increase this contrast, each image is processed by subtracting the values of the previous image from it. This has the effect of creating a dark band on the liquid side of the interface and a bright band on the solid side of the interface.

The microstructural features were extracted by first identifying the solid-liquid boundary for each of the cells and dendrites at the solidification front. These boundaries were converted to $(x, y)$-coordinates. The fitting of these coordinate tables provided the tip position and shape. Within each image, the tip positions were used to calculate the primary spacing. The tip radii were fitted with a second order parabola up to a distance of $\rho$ behind the tip location, in order to eliminate shape distortions from branching. This tip radius fitting up to a distance of one tip radius was also chosen to be consistent with the processing procedures used for the phase-field simulations (see Fig. 5), thus permitting a direct quantitative comparison between results from simulations and experiments.

\section{Simulations}

\subsection{Quantitative PF model for dilute binary alloy directional solidification}

We use a quantitative phase-field model for directional solidification presented elsewhere [73, 74] and also described in the attached Supplementary Material. The model is made quantitative by the use of the solute anti-trapping current [75] within the thin interface limit [73, 76], with no solid state species diffusion. It has been validated by several experimental comparisons under predominantly diffusive transport conditions $[34,40,41,45,73,74]$.

Assuming a constant cooling rate (or equivalent pulling velocity $V_{p}$ ) and an imposed temperature gradient $G$ aligned with the $x$ spatial direction, the model considers a frozen temperature profile $T=T_{0}+G(x-V t)$, with $T_{0}$ a reference temperature, here the alloy solidus temperature. 
We use a nonlinear preconditioning of the traditional phase field $\varphi$ of value $+1(-1)$ in the solid (liquid), which is replaced by the preconditioned phase field $\psi$ with

$$
\varphi(x, y, z, t) \equiv \tanh \left\{\frac{\psi(x, y, z, t)}{\sqrt{2}}\right\} .
$$

Solving the reformulated equations for $\psi$ enhances numerical stability for larger grid spacings [77].

We model microscopic thermal fluctuations by adding random noise on the $\psi$ field (see Supplementary Materials and Ref. [74]). The amplitude of the noise was verified to generate similar sidebranching dynamics as using a more quantitative formulation of noise $[45,78]$.

Additionally, we force a liquid layer to systematically wet the sides of the crucible in order to match experimental observations and avoid stabilizing half cells/dendrites within the sample thickness $H=200 \mu \mathrm{m}$. To do so, we impose a contact angle of the solid-liquid interface at the top and bottom grid points, i.e. at $z=0$ and $z=H$. While a more quantitative approach of boundaries wetting is possible [79], an accurate morphological description of these contact angles is not crucial in our experiments, as the affected junctions are located deep within the mushy zone, far away from the current zone of interest. This simplified approach allows us to integrate wetting conditions in a computationally efficient manner, and was already shown to produce quantitative three-dimensional simulations of thin-sample directional solidification experiments of transparent organic compounds [80].

The fundamental equations of the model, i.e. two coupled partial differential equations, are solved using finite differences on a regular grid of cubic elements of dimension $\Delta x$ and a Euler explicit time scheme with a time step $\Delta t=0.9 \Delta x^{2} /(6 D)$. The model is implemented for parallel calculations on Graphic Processing Units (GPU). The position of the most advanced solid phase is kept at a fixed location in the domain by tracking its position in time and shifting the domain accordingly.

\subsection{Convection modeling}

\subsubsection{Diffusive boundary-layer approach}

Convection can be introduced in phase-field simulations by direct coupling with Navier-Stokes equations using a no-slip condition at the solid-liquid interface [81]. However, this approach is computationally very costly especially in 3D. Consequently, we use a simplified boundary-layer approximation that provides a computationally tractable model of the effect of solute convection. We consider a 1D solute boundary layer (also sometimes referred to as a stagnant film) next to the solid-liquid interface [82, 83]. Within this boundary layer $(\mathrm{BL})$, solute transport is assumed to be diffusive, while outside of this BL, the solute is assumed to be well mixed and homogenized due to convection. Thus, a narrower BL thickness mimics the effect of stronger convection.

First introduced by derivation of an analytical solution to the Navier-Stokes equations in an idealized configuration [84], this BL approach has been extensively used to study the effect of convection on morpho- 
logical stability [85, 86, 87], dendritic growth kinetics [66, 88, 89, 90, 91], Bridgman solidification [82, 83, 92] including solidification of eutectic alloys [93], as well as complex formation of banded microstructures in peritectic alloys [94, 95].

\subsubsection{Integration into the PF model}

We include the BL approach in our phase-field model by setting a distance to the solid-liquid interface $\delta$ beyond which the liquid is at the nominal concentration $c_{\infty}$. Decreasing the value of $\delta$ then mimics a stronger convection in the melt.

We take advantage of the nonlinear preconditioning of the phase field variable in our simulations, following Eq. (1), as illustrated in Fig. 2. Since the steady state solution for a 1D diffuse interface propagating at a velocity $V_{0}$ has the from $\varphi \sim \tanh \left\{\left(x-V_{0} t\right) / W\right\}$ (see e.g. [77]), the change of variable with $\varphi \equiv \tanh (\psi / \sqrt{2})$ makes the preconditioned phase field $\psi$ proportional to a signed distance function. We use that property of $\psi$ to generalize the BL approach to non-planar morphologies, thus setting $c=c_{\infty}$ for each point beyond a distance defined by a threshold $\psi=\psi^{*}$.

This is illustrated in Fig. 3 for $G=50 \mathrm{~K} / \mathrm{cm}$ and $V=10 \mu \mathrm{m} / \mathrm{s}$. Fig. 3a and b respectively show the composition profile and the $\psi$ field in the $x$-direction in front of a dendrite tip, i.e. along the $(y, z)=$ $\left(y_{t i p}, z_{t i p}\right)$ line, with $\left(x_{t i p}, y_{t i p}, z_{t i p}\right)$ the coordinates of the dendrite tip. Using $\psi(x)$ from Fig. 3b, one can identify the threshold value $\psi=\psi^{*}$ that corresponds to a distance $\delta$ from the interface in the liquid, and then impose $c=c_{\infty}$ in the liquid for $x>\delta$, i.e. for $\psi<\psi^{*}$. Fig. 3a shows the resulting composition profiles for $\delta=100,150,200,400 \mu \mathrm{m}$, and $\delta \rightarrow \infty$ (i.e. a fully diffusive regime). Fig. 3c illustrates that isovalues of $\psi$ on a plane $\left(z=z_{t i p}\right)$ provide a good estimation of the three-dimensional distance to the interface, here represented for a purely diffusive simulation.

\subsection{Simulations}

\subsubsection{Parameters}

We perform phase-field simulations using dilute $\mathrm{Al}-\mathrm{Cu}$ alloys parameters from the literature, summarized in Table 1. While values of the partition coefficient $k$ and the Gibbs-Thomson coefficient $\Gamma$ are fairly well known (see [28, 99, 100] and [28, 97], respectively), different values can be found for the liquid diffusion coefficient $D$, the liquidus slope $m$, and the interface anisotropy strength $\epsilon_{4}$. For the diffusion coefficient, we found the best agreement between simulations and experiments using $D \approx 2.410^{-9} \mathrm{~m}^{2} / \mathrm{s}$ as measured by Trivedi and co-workers from thin tube experiments [96]. For the liquidus slope, values given in the classic textbook by Kurz and Fisher [28] range from $m \approx-2.6 \mathrm{~K} / \mathrm{wt} \% \mathrm{Cu}$ at $c_{\infty}=2 \mathrm{wt} \% \mathrm{Cu}$ to $-4.9 \mathrm{~K} / \mathrm{wt} \% \mathrm{Cu}$ at higher eutectic composition, and an average slope between the melting point of pure aluminum and the eutectic triple point $[99,100]$ yields $m \approx-3.4 \mathrm{~K} / \mathrm{wt} \% \mathrm{Cu}$. We used an intermediate value $m=-3.0 \mathrm{~K} / \mathrm{wt} \% \mathrm{Cu}$. 
For the anisotropy strength, we used the value calculated from the mapping of the interfacial free energy using molecular dynamics simulations, yielding $\epsilon_{4}=0.012$ for an aluminum crystal [98].

While we explore different temperature gradients in the experiments, simulations exclusively focus on $G=50 \mathrm{~K} / \mathrm{cm}$ and a velocity range from $V=5$ to $50 \mu \mathrm{m} / \mathrm{s}$. Although it is likely that the strength of convection depends upon the growth velocity, as a first approximation we explore microstructure selection as a function of $V$ for fixed values of the diffusive BL thickness $\delta$.

Detailed numerical parameters resulting from preliminary convergence tests for each set of parameters appear in the Supplementary Material.

\subsubsection{Morphological stability}

We test the conditions of stability of a planar interface for different values of the diffusive boundary layer thickness $\delta$.

To do so, we use a simulation domain of cross-section $200 \times 200 \mu \mathrm{m}^{2}$ in $y \times z$, using symmetric (i.e. no-flux) boundary conditions in all directions. The initial interface, located at the liquidus temperature, is quasi-planar with a small random initial perturbation. We perform these simulations without noise, and track the amplitude of the interface shape, $\Delta x_{i}=x_{t i p}-x_{\text {groove }}$, between its maximum and minimum locations in the $x$-direction, respectively $x_{t i p}$ and $x_{\text {groove }}$. This amplitude tends to $\Delta x_{i}=0$ as $t \rightarrow \infty$ for a stable interface, and to $\Delta x_{i} \neq 0$ for an unstable interface (see Fig. 9b).

Since destabilization (or re-stabilization to a planar front) can occur slowly, those calculations are performed for a relatively long time of $50000 \mathrm{~s}$. The typical running time for each simulation spans from $35 \mathrm{~h}$ (for $\delta=50 \mu \mathrm{m}$ and $V \approx 3.19 \mu \mathrm{m} / \mathrm{s}$ ) to $61 \mathrm{~h}$ (for $\delta=400 \mu \mathrm{m}$ and $V \approx 2.44 \mu \mathrm{m} / \mathrm{s}$ ), performed on a single Nvidia Tesla K40c GPU.

\subsubsection{Spatially extended simulations}

For each velocity listed in Table 1, we run spatially extended simulations over domains of at least $2 \mathrm{~mm}$ in the $y$-direction (namely between 2003 and $2008 \mu \mathrm{m}$ depending on $V$ ), with $H=200 \mu \mathrm{m}$ in $z$ (see Supplementary Material for details). We perform those simulation for a BL thickness $\delta=100,150,200$, $400 \mu \mathrm{m}$ and for $\delta \rightarrow \infty$. Those simulations start with a planar interface at the liquidus temperature, and boundary conditions are set to symmetric (no-flux) in the $x$-direction, periodic in the $y$-direction, and imposing a wetting angle with the sample walls in the $z$-direction.

In the presence of liquid convection, i.e. with a lower $\delta$, simulations take longer to reach a homogeneous microstructure, so we performed them for a longer time, as listed in Table 2.

The typical running time for these simulations is between 3 days (for $\delta=200 \mu \mathrm{m}$ and $V=5 \mu \mathrm{m} / \mathrm{s}$ ) and 28 days (for $\delta=100 \mu \mathrm{m}$ and $V=50 \mu \mathrm{m} / \mathrm{s}$ ) on a single Nvidia Tesla K40c GPU. 


\subsubsection{Spacing stability range}

To identify the stability of a primary spacing $\Lambda$, we perform simulations of one half of a cell/dendrite within a cross-section size $(\Lambda / 2 \times H)$ in $(y \times z)$ (see Sec. 1.6 and Fig. 1 of the Supplementary Material). Boundary conditions are symmetric (no-flux) along the $x$ and $y$ boundaries, with an imposed wetting angle along the $z$ boundaries. As in Ref. [41], but keeping a fixed size $H$ in $z$, we progressively increase the size of the domain in $y$ until the half dendrite becomes unstable with respect to sidebranching, thus locating the upper boundary of the spacing stability range $\Lambda_{\max }$. Simulated times were taken long enough for the half cell/dendrite to stabilize at a given undercooling, typically from 3 to 25 minutes, respectively for $V=50$ and $5 \mu \mathrm{m} / \mathrm{s}$ (see Supplementary Material for further details).

Similarly, we shrink the domain in $y$ to find stable (or metastable) states at smaller $\Lambda$. We then duplicate the resulting steady states by doubling the size of the domain in $y$, and starting a simulation with two halves of cells facing each other, hence testing the spacing stability with respect to elimination to determine the lower limit of spacing stability $\Lambda_{\min }$. Starting from a perfectly symmetrical configuration, the elimination process can occur slowly, and therefore these simulations were performed for a relatively long time, namely up to $t=200 \mathrm{~min}$.

We perform such calculations of $\Lambda_{\min }$ and $\Lambda_{\max }$ for thicknesses of the diffusive boundary layer $\delta=100$, $150,200 \mu \mathrm{m}$ as well as for a fully diffusive liquid domain (i.e. $\delta \rightarrow \infty$ ).

Running times for these simulations typically span from $75 \mathrm{~min}$ (for one half of a cell at $V=10 \mu \mathrm{m} / \mathrm{s}$, $\delta=100 \mu \mathrm{m}$, and $\Lambda \approx 96 \mu \mathrm{m}$ over $10 \mathrm{~min}$ ) to 8 days (for two halves of cells at $V=5 \mu \mathrm{m} / \mathrm{s}, \delta \rightarrow \infty$, and $\Lambda \approx 328 \mu \mathrm{m}$ over $200 \mathrm{~min}$ ) on a single Nvidia GTX680 GPU. For each set of parameters, building the entire stable branch from $\Lambda_{\min }$ to $\Lambda_{\max }$ required from about 10 to 30 individual simulations.

\subsection{Results post-processing}

For each simulation, we extract the primary tip locations, the primary dendritic spacings, and the primary tip radii using procedures further detailed in the attached Supplementary Material, and briefly summarized here. For consistency with experimental measurements, all spacings are measured parallel to the $y$-direction.

It is useful to distinguish morphological features within different regions, depending upon whether these regions exhibit one or two rows of cells or dendrites within the sample thickness. This is illustrated in Fig. 4, showing a snapshot of the microstructure at $t=600 \mathrm{~s}$ in the spatially extended simulation for $G=50 \mathrm{~K} / \mathrm{cm}$, $V=20 \mu \mathrm{m} / \mathrm{s}$ and $\delta=100 \mu \mathrm{m}$. Based on the angle $\theta$ between two adjacent tips from the $y$-direction in the $(y, z)$ plane (Fig. $4 \mathrm{~b}$ ), a dendrite is regarded as part of the two-row region if $|\theta|>\theta^{*}$ with both its neighboring dendrites, using a threshold value $\theta^{*}=20^{\circ}$. Post-processing scripts automatically generate colored dendrite tips in red if they belong to the two-row region and blue otherwise, as illustrated in Fig. 4 and other figures throughout the paper. 
Hence, we identify features within the one-row region using a subscript " $1 r$ " and within the two-row region using a subscript "2r" (Fig. 4b). The corresponding symbol, e.g. $\Lambda$, without any subscript refers to the global average. The minimum and maximum spacings measured over the whole array are respectively $\Lambda_{\text {low }}$ and $\Lambda_{\text {high }}$, which should not be confused with the limits of the spacing stability range for individual dendrites, $\Lambda_{\min }$ and $\Lambda_{\max }$.

To extract primary dendrite tip radii, we tabulate the location of the intersection of the solid-liquid interface with the planes $\left(y=y_{t i p}\right)$ and $\left(z=z_{t i p}\right)$ for each tip. These intersections are illustrated, with red and blue lines respectively, in Fig. 5 a for the simulation with $G=50 \mathrm{~K} / \mathrm{cm}, V=15 \mu \mathrm{m} / \mathrm{s}$ and $\delta \rightarrow \infty$.

Then, in the $y$-direction, we extract the tip radius $\rho_{y}$ by fitting the interface shape $y_{i}(x)$ within the $\left(z=z_{t i p}\right)$ plane to a parabola $x=x_{t i p}-\left(y_{i}-y_{t i p}\right)^{2} /\left(2 \rho_{y}\right)$. We proceed similarly to fit each intersection $z_{i}(x)$ within each $\left(y=y_{\text {tip }}\right)$ plane and obtain the tip radius in the $z$-direction $\rho_{z}$. Since fitted tip radii may depend on the fitting range, we choose to select the radius that corresponds to a fit until a distance of one radius behind the tip location, thus remaining consistent with experimental measurements. To do so, we fit the radius over different ranges and select the fit that has a fitting distance closest to the corresponding fitted radius, as illustrated in Fig. 5d for the leftmost dendrite in Fig. 5 (see details in the attached Supplementary Material). We then calculate the radius of mean curvature $\rho$ of each dendrite with $1 / \rho=\left(1 / \rho_{y}+1 / \rho_{z}\right) / 2$.

\section{Results and discussion}

\subsection{Overall microstructure selection}

First, we present a qualitative overview of observed microstructures in both experiments and simulations. Fig. 6 shows X-ray radiographs from the directional solidification experiments for two temperature gradients $(G=50$ and $80 \mathrm{~K} / \mathrm{cm})$ at various growth velocities, showing typical planar, cellular, and dendritic microstructures (with increasing $V$ from top to bottom). On the simulation side, Fig. 7 shows phase-field results for a fixed temperature gradient $G=50 \mathrm{~K} / \mathrm{cm}$, growth velocities from $V=5$ to $50 \mu \mathrm{m} / \mathrm{s}$ for a fully diffusive regime $(\delta \rightarrow \infty)$ and different BL thicknesses $\delta=200 \mu \mathrm{m}$, and $100 \mu \mathrm{m}$.

The dilute alloy concentration $(\mathrm{Al}-1.4 \mathrm{wt} \% \mathrm{Cu}$, i.e. Al-0.6at.\% Cu) leads to a weak absorption contrast in the images, since X-ray absorption is directly related to the local density and average atomic number. More defined images could be obtained using a more concentrated Al-Cu alloy (see e.g. [26, 101]). However, it is more practical to achieve planar growth and explore morphological transitions using a dilute alloy. More concentrated alloys also tend to exhibit much sharper dendritic microstructures composed of needle-like branches (see e.g. [101]), which makes them more challenging to simulate quantitatively, since phase-field requires a good numerical description of the tip radius morphology, i.e. a small grid spacing $\Delta x$. (For instance, doubling the nominal concentration to $c_{\infty}=2.8 \mathrm{wt} \% \mathrm{Cu}$ would result in simulations almost six times slower, as explained in Section 2.8 of the Supplementary Material.) Therefore, we purposely selected 
a dilute alloy that provides a reasonable compromise between X-ray imaging contrast and accessibility to quantitative 3D phase-field calculations.

In terms of image processing, the weak absorption contrast makes an accurate measurement of tip radii more difficult, but does not significantly affect the primary spacing measurements that appear clearly on every image. Yet, as discussed in Sec. 4.3, in some images it is difficult to make definitive conclusions about whether the microstructure consists of a unique row or two rows of cells or dendrites, particularly in the cellular regime or close to the cell-to-dendrite transition (e.g. for $G=80 \mathrm{~K} / \mathrm{cm}$ and $V=5.5 \mu \mathrm{m} / \mathrm{s}$ in Fig. 6 ). The weak absorption contrast also makes it impossible to map the solute content in the liquid, and hence to bring evidence of convective transport in the liquid.

Fig. 7 shows the significant influence of the boundary layer width $\delta$ on selected microstructures at low growth velocity. The thick orange line in the background schematically illustrates the transition from a mostly diffusive transport regime when $D / V<\delta$ to a mostly convective regime when $\delta<D / V$. This transition occurs progressively when the BL width $\delta$ is of the same order as the natural diffusion length $D / V$. The microstructures most strongly affected by the extent of liquid convection are those within the $\delta<D / V$ domain on the top-right of Fig. 7 .

Fig. 7 also shows microstructures involving two rows of cells/dendrites for $V \leq 10 \mu \mathrm{m} / \mathrm{s}$ and mixed microstructures with both one-row and two-row regions for other velocities when $\delta=100 \mu \mathrm{m}$. While the microstructures exclusively composed of two rows within the sample thickness are in a stable steady state, we show in Sec. 4.3.1 that mixed microstructures (here for $\delta=100 \mu \mathrm{m}$ and $V=15$ or $50 \mu \mathrm{m} / \mathrm{s}$ ) are still evolving very slowly toward a one-row microstructure.

In the following subsections, we make a direct comparison of experimental measurements with phasefield predictions, and show that a quantitative agreement can only be achieved by including the effect of convective transport in the liquid.

\subsection{Morphological selection}

We focus first on morphological transitions between planar, cellular, and dendritic growth regimes. Fig. 8 shows the selection map from experiments. Our criteria for distinguishing cells from dendrites is respectively the absence or presence of secondary sidebranches.

The observed planar-to-cellular threshold (solid green line) is shifted toward higher velocities than expected from classical morphological stability theory (thick black solid line). Using the constitutional undercooling criterion [30], the critical velocity $V_{c}$ for a planar interface destabilization is reached when $(\partial c / \partial x)_{i}=(\partial c / \partial T)_{i}(\partial T / \partial x)_{i}=G / m$ on the liquid side of the interface (denoted by the subscript $\left.i\right)$. The constitutional undercooling limit for planar instability is thus at

$$
V_{c}=\frac{G D k}{m c_{\infty}(k-1)}
$$


which for instance gives a critical velocity $V_{c} \approx 0.465 \mu \mathrm{m} / \mathrm{s}$ for $G=50 \mathrm{~K} / \mathrm{cm}$. Yet, our experiments clearly show a limit between planar and cellular growth at $V \approx 3.0 \mu \mathrm{m} / \mathrm{s}$ for this temperature gradient. The cell-to-dendrite transition is similarly shifted toward a velocity of approximately 4 times the measured planar-to-cell transition velocity.

The planar stability at higher growth velocity is a first indication of convective transport in the liquid. As illustrated by the composition profiles in Fig. 3a, the strength of liquid convection affects the value of the equilibrium concentration on the liquid side of the interface, and hence the concentration gradient $(\partial c / \partial x)_{i}$. Thus, the constitutional undercooling limit $(\partial c / \partial x)_{i}=G / m$ in presence of a boundary layer of width $\delta$ follows (see analytical derivation in the Supplementary Material)

$$
\delta=-\frac{D}{V_{c}} \ln \left(\frac{k}{k-1}-\frac{m c_{\infty}}{D G} V_{c}\right) .
$$

Hence, stronger convection in the melt (i.e. a lower $\delta$ ) yields an increase of $V_{c}$, hence stabilizing the planar interface at higher growth velocities.

The experimentally assessed $V_{c}$ suggests that convection plays a significant role in our experiments. This is consistent with early experiments by Grange and collaborators reporting an increase of $V_{c}$ due to liquid convection in a dilute $\mathrm{Al}-\mathrm{Cu}$ alloy [52], and recent experiments showing further evidence of convection in thin-sample $\mathrm{Al}-\mathrm{Cu}$ alloys using in situ $\mathrm{X}$-ray radiography [62, 63, 64].

An additional way to illustrate the influence of liquid convection is to compare the results from similar experiments growing downward from the top, rather than upward from the bottom. With the rejected copper solute heavier than the average liquid, the growth from the top downward produces stronger convective currents $[25,58,62,63]$, and consequently a planar stability at even higher growth velocities. Data points in larger full symbols in Fig. 8 correspond to experiments with such a downward growth. In these experiments, morphological transitions appear further shifted to higher velocities, thus supporting our hypothesis regarding the strong effect of liquid convection.

To validate Eq. (3) and obtain a first estimation of an appropriate value for the BL thickness $\delta$, we perform PF calculations described in Sec. 3.3.2. For $G=50 \mathrm{~K} / \mathrm{cm}$, we test the stability of a planar interface for values of $V$ just below and just above the theoretical stability limit $V_{c}$, namely for $V=V_{c} \pm 0.05 \mu \mathrm{m} / \mathrm{s}$ for $\delta=50,100,150,200,300$, and $400 \mu \mathrm{m}$, with $V_{c}=3.14,2.99,2.86,2.74,2.54$, and $2.39 \mu \mathrm{m} / \mathrm{s}$, respectively, according to Eq. (3). Simulations results, in Fig. 9a, show up-pointing triangles for a stable planar interface, i.e. with $\Delta x_{i} \rightarrow 0$ (dotted lines in Fig. 9b), and down-pointing triangles show unstable interfaces, i.e. with $\Delta x_{i}$ increasing to a finite value (solid lines in Fig. 9b).

These results, in good agreement with Eq. (3) (red solid line in Fig. 9a), also give a first estimation of $\delta$ representative of the strength of convection in our experiments. A value of $\delta \approx 100 \mu \mathrm{m}$ appears to yield transport conditions in best agreement with our experiments, with $V_{c} \approx 3 \mu \mathrm{m} / \mathrm{s}$. We show in the following subsections that $\delta \approx 100 \mu \mathrm{m}$ also yields a good agreement with experiments in terms of selected 
microstructural features.

\subsection{Selection of primary spacing}

Fig. 10 shows, for each experiment, the average value of the measured primary spacing (symbols) and the spacing distribution range $\left[\Lambda_{\text {low }}, \Lambda_{\text {high }}\right]$ (uncertainty limits). We only show the results for $V \geq 5 \mu \mathrm{m} / \mathrm{s}$ to discard possible deviations due to the proximity of the planar-to-cellular transition.

The increase of spacing with growth velocity is in sharp disagreement with classical theory that predict a power law $\Lambda \sim V^{\alpha}$, typically with $\alpha=-0.25$ in the dendritic regime $[13,53]$. In contrast, our measurements yield $\alpha \approx 0.5$, as illustrated by the fit to $V^{0.5}$ for measurements at $G=50$ and $65 \mathrm{~K} / \mathrm{cm}$ in dotted gray line in Fig. 10.

In the following subsections, we show that this inversion of the $\Lambda(V)$ trend is an effect of strong convection in the liquid, which induces: (i) a very slow evolution toward a homogeneous microstructural state, suggesting that observed microstructure might still be slowly evolving (Sec. 4.3.1); and (ii) an a reduction of the interaction distance between dendrites to a length $\delta$ instead of the typical diffusion length $D / V$ (Sec. 4.3.2 and 4.3.3).

\subsubsection{Transient microstructural evolution}

As illustrated in Fig. 7, some predicted microstructures exhibit a mixture of one-row and two-row regions, even after the simulated times listed in Table 2. We found that such simulations have not yet reached a microstructural steady state, and are slowly evolving toward a one-row structure.

We illustrate this evolution with the spatially-extended simulation at $G=50 \mathrm{~K} / \mathrm{cm}, V=20 \mu \mathrm{m} / \mathrm{s}$, and $\delta=100 \mu \mathrm{m}$. Figure 11a shows the time evolution of primary spacings (defined in Fig. 4b) in this simulation. Figure 11b-i illustrates how the interface pattern evolves slowly from two rows of dendrites (b) to a homogeneous microstructure with one row of dendrites (i) within the thickness of the sample, as seen from the liquid side.

At the beginning of the simulation, the planar interface recoils within the temperature gradient and its undercooling increases. The interface undergoes morphological instability at $t \approx 20 \mathrm{~s}$, and the front accelerate to stabilize at a dimensionless undercooling of $\Delta \approx 0.345$. Slightly before $t=60 \mathrm{~s}$, the dendritic front reaches its steady state velocity and then stays within $\pm 1 \%$ of $V=20 \mu \mathrm{m} / \mathrm{s}$ until the end of the simulation.

Yet, Fig. 11 shows that the microstructure still evolves significantly and only reaches a homogeneous steady state at $t \geq 1500 \mathrm{~s}$. During this slow primary spacing coarsening, solutal interactions among dendrites lead to progressive elimination events in the two-row region, such that the array slowly evolves toward a unique row of dendrites within the sample thickness. This behavior is a clear indication of bi-stability, with a dynamically favored stable state (with one row) propagating within a metastable state (with two 
rows). Thus, the coarsening of the microstructure stems from a mechanism of propagation-invasion rather than from elimination events occurring homogeneously throughout the two-row region. This mechanism is three-dimensional in nature, and hence depends strongly on the sample confinement. It occurs at a much slower time scale than the initial transient spacing coarsening step, predicted so far with two-dimensional simulations $[102,103,104]$.

In Fig. 11a, the smallest spacing $\Lambda_{\text {low }}(\triangle)$ and largest spacing $\Lambda_{\text {high }}(\nabla)$ in the array exhibit jumps linked to individual elimination events. In the meantime, the average primary spacing $\Lambda$ (०) evolves step by step from the spacing in the two-row region $\Lambda_{2 \mathrm{r}}(\diamond)$ to the spacing in the one-row region $\Lambda_{1 \mathrm{r}}(\square)$. Interestingly, both $\Lambda_{1 \mathrm{r}}$ and $\Lambda_{2 \mathrm{r}}$ exhibit limited variations during the coarsening process, as they reach a value close to their final value before $t=400 \mathrm{~s}$. This means that as soon as the one-row region has reached a population of four to five dendrites (i.e. Fig. 11d at $t \approx 386 \mathrm{~s}$ ), one can approximate the final steady state spacing as $\Lambda(t \rightarrow \infty) \approx \Lambda_{1 \mathrm{r}}$. In the specific case of Fig. 11, approximating $\Lambda(t \rightarrow \infty)$ with $\Lambda_{1 \mathrm{r}}(t \approx 400 \mathrm{~s})$ would lead to a relative error of less than $4 \%$.

Other simulations that result in a heterogeneous microstructure undergo a similar slow coarsening mechanism, which we only observed for $\delta \leq 150 \mu \mathrm{m}$, i.e. for strong liquid convection (Fig. 7). This transient microstructural evolution is too slow to capture in the experimentally observed time frame, since the front sweeps through the $1.7 \mathrm{~mm}$ field of view in about from 30 seconds to 6 minutes for respectively $V=50$ to $5 \mu \mathrm{m} / \mathrm{s}$. Due to the limited image contrast in such a dilute alloy, it is possible that what initially appeared to be a unique periodic array of dendrites at low velocity was actually composed of two rows of dendrites, which are still evolving toward a homogeneous microstructure.

\subsubsection{Spacings in spatially-extended simulations}

Fig. 12 shows $\Lambda_{1 \mathrm{r}}$ and $\Lambda_{2 \mathrm{r}}$ as a function of $V$ for different $\delta$ at the end of each spatially extended simulation (i.e. after simulated times in Table 2).

Some fluctuation in the selected spacing can be attributed to a quantization effect, since a finite number of spacings have to be selected within the $2 \mathrm{~mm}$ periodic domain in $y$. These fluctuations appear clearly at $V=10 \mu \mathrm{m} / \mathrm{s}$ and $\delta=400 \mu \mathrm{m}$, or at $V=15 \mu \mathrm{m} / \mathrm{s}$ and $\delta=150 \mu \mathrm{m}$.

The shaded regions behind each curve in Fig. 12 represent the range of spacings $\left[\Lambda_{\text {low }}, \Lambda_{\text {high }}\right]$ within the array. Except for two simulations, namely with $\delta=100 \mu \mathrm{m}$ and $V=15$ or $50 \mu \mathrm{m} / \mathrm{s}$ that have not yet reached a complete microstructural steady state (see Sec. 4.3.1), the spacing distribution range are relatively narrow.

As already shown qualitatively in Fig. 7, convection has a significant effect on selected spacings. Stronger convection, i.e. a lower $\delta$, induces a lower spacing, particularly at low velocities. Convection actually reduces the diffusive boundary layer around dendrites, thus preventing interactions between dendrites at a length scale much larger than $\delta$. For $\delta=100 \mu \mathrm{m}$, this decrease of spacing at $V<15 \mu \mathrm{m} / \mathrm{s}$ also comes with 
a transition to a steady two-row microstructure with an even lower spacing $\Lambda_{2 \mathrm{r}}$. In contrast to mixed microstructures discussed in Sec. 4.3.1, these low velocities at $\delta=100 \mu \mathrm{m}$ actually yield a stable steady microstructure exclusively composed of two rows.

For high velocities, spacings in mixed convective-diffusive conditions converge toward the spacing evolution in the fully diffusive case $(\delta \rightarrow \infty)$, closely following $\Lambda \sim V^{-1 / 2}$ (gray dotted line), i.e. with $\partial \Lambda / \partial V<0$.

The transition from positive to negative $\partial \Lambda / \partial V$ happens when the natural diffusion length $D / V$ becomes lower than the imposed BL thickness $\delta$. According to classical theory, the maximum of $\Lambda(V)$ corresponds to the cell-to-dendrite transition [51, 52]. However, we show later in Sec. 4.4 that the observed microstructures unambiguously behave like dendrites, with a constant $\rho^{2} V$ even in the region where $\Lambda$ increases with $V$.

The scatter in experimental measurements for $\Lambda$ at $V=15 \mu \mathrm{m}$ in Fig. 12 seems to match the scatter between simulated $\Lambda_{1 \mathrm{r}}$ and $\Lambda_{2 \mathrm{r}}$, which might be an additional indication that some measurements were performed on two rows of dendrites or on inhomogeneous microstructures (see Sec. 4.3.1).

The best agreement with experimental measurements (black crosses in Fig. 12) corresponds to $\delta$ between 100 and $150 \mu \mathrm{m}$, further supporting our interpretation that strong liquid convection is responsible for the unexpected increase of spacing with velocity.

\subsubsection{Stable spacing range}

Figure 13 shows the primary spacing range stability limits $\Lambda_{\max }$ (a) and $\Lambda_{\min }$ (b) as a function of the growth velocity $V$ for different values of $\delta$. (These should not be confused with $\Lambda_{\text {low }}$ and $\Lambda_{\text {high }}$ that are the minimum and maximum spacings selected within the spatially extended simulations.) The dependence of these ranges on $\delta$ is similar to what is observed for average spacings within spatially extended simulations. For finite values of $\delta$, both $\Lambda_{\min }$ and $\Lambda_{\max }$ exhibit an increase with $V$ at low velocities, followed by a decrease at higher $V$, as their evolution approaches the purely diffusive behavior.

While we could not find any one-row stability region at $V<10 \mu \mathrm{m} / \mathrm{s}$ for $\delta=100 \mu \mathrm{m}$, the experimental measurements at $G=50 \mathrm{~K} / \mathrm{cm}$ mostly fall within the corresponding stability range for $V \geq 10 \mu \mathrm{m} / \mathrm{s}$, and they are also partially within the stability range for $\delta=150 \mu \mathrm{m}$. This is, once again, an unambiguous illustration of the crucial role of strong convection in the liquid phase.

In a fully diffusive transport regime both $\Lambda_{\min }(V)$ and $\Lambda_{\max }(V)$ are well fitted to a power law $\Lambda \sim V^{-1 / 2}$, shown by the dotted gray lines in Fig. 13. Hence, the average value of selected primary dendritic spacings (Fig. 12) and its upper and lower stability limits (Fig. 13) are all proportional to one another, as suggested by Hunt and $\mathrm{Lu}[35,27]$ and commonly assumed (e.g. [46, 61]).

In all simulations at $V \geq 10 \mu \mathrm{m} / \mathrm{s}$, the ratio $\Lambda_{1 \mathrm{r}} / \Lambda_{\min } \approx 1.35 \pm 0.25$, and the ratio $\Lambda_{\max } / \Lambda_{\min } \approx 3.10 \pm$ 0.40 , for all explored values of $\delta$. These results are consistent with recent simulations in the diffusive regime, using both phase-field calculations $[34,45]$ and multiscale dendritic needle network (DNN) modeling [47, 48]. In these simulations, we also found $\Lambda_{\max } / \Lambda_{\min }$ to be close to 3 or 4 . Yet, the dynamically selected spacings 
in spatially extended DNN simulations tend to systematically fall within the lower half of the stable range, hence yielding an average selected $\Lambda / \Lambda_{\min } \approx 1.5$. This explains why, even though the basic assumption that $\Lambda_{\max } / \Lambda_{\min } \approx 2$ seems incorrect, assuming an average spacing between $\Lambda_{\min }$ and $2 \Lambda_{\min }$ nonetheless yields a good approximation of experimentally measured spacings [35, 27].

This is also consistent with measurements in all of our experiments for $V \geq 10 \mu \mathrm{m} / \mathrm{s}$, with $\Lambda / \Lambda_{\text {low }} \approx$ $1.69 \pm 0.61$, and $\Lambda_{\text {high }} / \Lambda_{\text {low }} \approx 2.18 \pm 0.98$. However, as pointed out in Sec. 4.3.1, it is likely that our measurements occurred while the microstructure is still slowly evolving, thus yielding a wider distribution than expected in a microstructural steady state.

\subsection{Selection of primary tip radius}

It was shown as early as in the late 1980's that unless external flow is extremely strong, the dendrite tip selection parameter $\sigma \sim 1 /\left(\rho^{2} V\right)$ should not change [105], which was verified numerically [65, 66, 67, 68, 69, 70] as well as experimentally [71]. Therefore, even in presence of convection we do not expect a significant difference of tip radius with respect to theory in the diffusive regime, i.e. $\rho \sim V^{-1 / 2}$.

Fig. 14 shows the average value (symbols) and distribution range (uncertainty limits) of primary tip radii measured in our experiments. The distribution of radii is broader than theoretically expected, which can be attributed to the lack of contrast in images for such a dilute alloy (see Sec. 4.1), and possibly to measurements being performed on inhomogeneous microstructures due to convection (see Sec. 4.3.1). Yet, the average tip radii still essentially follows the power law $\rho \sim V^{-1 / 2}$ from solvability theory, as illustrated by the fit to $\rho \sim V^{-1 / 2}$ for $G=50$ and $65 \mathrm{~K} / \mathrm{cm}$ in dotted gray line in Fig. 14 .

The phase-field results for $G=50 \mathrm{~K} / \mathrm{cm}$ in Fig. 15 also agree with classical theory [105]. Only average values of selected radii are presented in Fig. 15, because the range of individual $\rho$ values in simulations is very narrow, i.e. of the same order as the size of the symbols. The mean tip radius $\rho$ (Fig. 15a) of all dendrites in the one-row region (open symbols) closely follows $\rho \sim V^{-1 / 2}$, regardless of $\delta$.

While the mean tip radius in Fig. 15a remains close to the fitted $\rho \sim V^{-1 / 2}$, the radii in the $y$ and the $z$-direction, respectively in Fig. $15 \mathrm{~b}$ and c, slightly deviate from this fit at low velocities. This is an effect of the thin-sample confinement: dendrite tips become less axisymmetrical, with a larger radius in the $y$-direction parallel to the sample walls and a smaller radius in the $z$-direction normal to the walls, as e.g. illustrated for $V \leq 10 \mu \mathrm{m} / \mathrm{s}$ and $\delta \rightarrow \infty$ in Fig 7. However, even for such flat dendrites, the mean radius of curvature seems conserved as long as there is only one row of dendrites.

For low velocities and strong convection, the transition from one to two rows of dendrites (respectively open and full symbols in Fig. 15) occurs with a decrease in tip radii, as expected from relatively smaller microstructural features. This evolution at $V<10 \mu \mathrm{m} / \mathrm{s}$ and $\delta=100 \mu \mathrm{m}$ is close to that observed in the experiments, possibly supporting our interpretation that we might be observing two rows of dendrites for low velocity experiments. 
The effect of convection (i.e. of $\delta$ ) on tip radii remains small compared to its effect on primary spacings. This highlights the decoupling between the dendritic spacing selection that involves diffusive interactions at the scale of $D / V$ and the tip radius selection linked to curvature-induced diffusive fluxes along the side of a dendrite tip that occur at a scale close to $\rho$. While liquid convection may lead to a boundary layer thickness $\delta$ comparable or smaller than $D / V$, hence considerably affecting spacing selection, $\delta$ always remains larger than $\rho$, such that it barely affects the selected tip radii.

\section{Summary and Outlook}

We combined in situ X-ray observations of dilute $\mathrm{Al}-\mathrm{Cu}$ alloy directional solidification experiments with three-dimensional phase-field $(\mathrm{PF})$ simulations to shed light on dendritic microstructure selection mechanisms in thin sample experiments.

We proposed a simple way to qualitatively introduce the effect of liquid convection into phase-field calculations, by imposing a diffusive boundary layer (BL) in the vicinity of the solid-liquid interface. Thus, we presented evidence of strong liquid convection in the experiments.

Liquid convection has two major effects. Firstly, it increases the stability of a planar interface at higher velocities, shifting the planar-cellular and the cellular-dendritic transitions toward higher velocities. Secondly, convective effects are at the origin of the unexpected dependence of primary dendritic spacings $\Lambda$ upon growth velocity $V$ measured experimentally, with $\partial \Lambda / \partial V>0$, as opposed to classical theory in the diffusive regime.

Our simulations also show that convection in the liquid may lead to slow homogenization of the microstructure within the thin sample at the time scale of several tens of minutes, while the steady growth velocity is usually reached within about one minute (Fig. 11).

Regardless of solidification or convective conditions, the limits of the stable spacing range $\Lambda_{\min }$ and $\Lambda_{\max }$, and the average dynamically selected spacing are all proportional to one another as suggested by Hunt and $\mathrm{Lu}$ in the diffusive regime [35, 27]. However, our simulations lead to a ratio $\Lambda_{\max } / \Lambda_{\min } \approx 3$, in contrast to the common assumption $\Lambda_{\max } / \Lambda_{\min } \approx 2$ (see e.g $[46,61]$ ), but in agreement with other phase-field $[34,45]$ and multiscale dendritic growth simulations [47, 48]. Yet, in the present study like in previous simulations $[47,48]$, the average selected array spacings appear to systematically fall within the lower half of the stable spacing range. This explains the agreement between experimental measurements and the assumption that $\Lambda_{\max } / \Lambda_{\min } \approx 2$, as most spacings fall within the $\left[\Lambda_{\min }, 2 \Lambda_{\min }\right]$ range.

In terms of primary tip radius, while convection in our experiments tends to broaden the range of selected radii (Fig. 14), both experiments and simulations yield an average radius $\rho \sim V^{-1 / 2}$ with a weak effect of convection. This is consistent with microscopic solvability theory, exhibiting a weak dependence upon moderate convection as reported in earlier literature [65, 66, 67, 68, 71, 69, 70]. 
Finally, it seems worth stressing some limitations of the current study. The current BL approach relies on an approximate 1D description of convection effects in the melt, such that full 3D simulations of coupled solidification and fluid flow $[81,61,106]$ remain needed for further validation. We also made the assumption that the BL thickness $\delta$ was independent of the growth velocity $V$, while it seems likely that the extent of convection and the growth velocity are not independent.

Despite these limitations, the simple diffusive BL approach successfully identifies the presence of convection in the liquid phase as the key mechanism responsible for the shift of the onset of morphological instability to higher velocity and the change of slope of the dependence of primary spacing on velocity. While the shift of $V_{c}$ is a known effect of convection [85, 86, 87], the change of slope of the $\Lambda$ - $V$ relation is a novel aspect of the present work. The current study also highlights the importance of the sample thickness in the interpretation of two-dimensional radiography images, thereby motivating a more in depth analysis of the effect of sample geometry on microstructural pattern formation and the development of a directional solidification furnace enabling three-dimensional in situ tomography.

\section{Acknowledgements}

This study was supported by A.J.C.'s Early Career award from the U.S. DOE, Office of Basic Energy Sciences, Division of Materials Sciences and Engineering and Los Alamos National Laboratory, operated by Los Alamos National Security, LLC under Contract No. DE-AC52-06NA25396 from the U.S. Department of Energy. A.K. was supported by Grant No. DE-FG02-07ER46400 from the U.S. Department of Energy, Office of Basic Energy Sciences. Use of the Advanced Photon Source, an Office of Science User Facility operated for the U.S. DOE Office of Science by Argonne National Laboratory, was supported by the U.S. DOE under Contract No. DE-AC02-06CH11357.

\section{References}

[1] J.S. Langer, Lectures on the Theory of Pattern Formation, in: Chance and Matter (Les Houches, Session XLVI), J. Souletie, J. Vannimenus, R. Stora (Eds.), North-Holland, Amsterdam, 1987, pp. 629-711.

[2] J.S. Langer, Dendrites, Viscous Fingers, and the Theory of Pattern Formation, Science 243 (1989) $1150-1156$.

[3] A. Karma, D. Tourret, Curr. Opin. Solid State Mater. Sci. 20 (2016) 25-36.

[4] M. Rappaz, Modeling and characterization of grain structures and defects in solidification, Curr. Opin. Solid State Mater. Sci. 20 (2016) 37-45.

[5] W.J. Boettinger, S.R. Coriell, A.L. Greer, A. Karma, W. Kurz, M. Rappaz, R. Trivedi, Solidification microstructures: recent developments, future directions, Acta Mater. 48 (2000) 43-70.

[6] M. Asta, C. Beckermann, A. Karma, W. Kurz, R. Napolitano, M. Plapp, G. Purdy, M. Rappaz, R. Trivedi, Solidification microstructures and solid-state parallels: Recent developments, future directions, Acta Mater. 57 (2009) 941-971.

[7] K.A. Jackson, J.D. Hunt, Transparent compounds that freeze like metals, Acta Metall. 13 (1965) $1212-1215$.

[8] S.C. Huang, M.E. Glicksman, Overview 12: Fundamentals of dendritic solidification - I. Steady-state tip growth, Acta Metall. 29 (1981) 701-715 
[9] S.C. Huang, M.E. Glicksman, Overview 12: Fundamentals of dendritic solidification - II development of sidebranch structure, Acta Metall. 29 (1981) 717-734

[10] R. Trivedi, K. Somboonsuk, Constrained dendritic growth and spacing, Mater. Sci. Eng. 65 (1984) 65-74.

[11] K. Somboonsuk, R. Trivedi, Dynamical studies of dendritic growth, Acta Metall. 33 (1985) $1051-1060$.

[12] R. Trivedi, K. Somboonsuk, Pattern formation during the directional solidification of binary systems, Acta Metall. 33 (1985) 1061-1068.

[13] W. Kurz, D.J. Fisher, Dendrite growth at the limit of stability: tip radius and spacing, Acta Metall. 29 (1981) 11-20.

[14] K. Somboonsuk, J.T. Mason, R. Trivedi, Interdendritic spacing: Part I. experimental studies, Metall. Trans. A 15 (1984) 967-975.

[15] H. Esaka, W. Kurz, Columnar dendrite growth: experiments on tip growth, J. Cryst. Growth 72 (1985) 578-584.

[16] J.D. Verhoeven, J.T. Mason, R. Trivedi, The effect of convection on the dendrite to eutectic transition, Metall. Trans. A 17 (1986) 991-1000.

[17] S.H. Han, R. Trivedi, Primary spacing selection in directionally solidified alloys, Acta Metall. Mater. A 42 (1994) $25-41$.

[18] W. Losert, B.Q. Shi, H.Z. Cummins, J.A. Warren, Spatial period-doubling instability of dendritic arrays in directional solidification, Phys. Rev. Lett. 77 (1996) 889.

[19] R.H. Mathiesen, L. Arnberg, F. Mo, T. Weitkamp, A. Snigirev, Time resolved x-ray imaging of dendritic growth in binary alloys, Phys. Rev. Lett. 83 (1999) 5062.

[20] H. Yasuda, I. Ohnaka, K. Kawasaki, A. Sugiyama, T. Ohmichi, J. Iwane, K. Umetani, Direct observation of stray crystal formation in unidirectional solidification of Sn-Bi alloy by X-ray imaging, J. Cryst. Growth 262 (2004) 645-652.

[21] H. Nguyen-Thi, L. Salvo, R.H. Mathiesen, L. Arnberg, B. Billia, M. Suery, G. Reinhart, On the interest of synchrotron X-ray imaging for the study of solidification in metallic alloys, C. R. Phys. 13 (2012) 237-245.

[22] R.H. Mathiesen, L. Arnberg, K. Ramsøskar, T. Weitkamp, C. Rau, A. Snigirev, Time-resolved x-ray imaging of aluminum alloy solidification processes, Metall. Mater. Trans. B 33 (2002) 613-623.

[23] H. Nguyen-Thi, H. Jamgotchian, J. Gastaldi, J. Härtwig, T. Schenk, H. Klein, B. Billia, J. Baruchel, Y. Dabo, Preliminary in situ and real-time study of directional solidification of metallic alloys by x-ray imaging techniques, J. Phys. D Appl. Phys. 36 (2003) A83.

[24] R.H. Mathiesen, L. Arnberg, X-ray radiography observations of columnar dendritic growth and constitutional undercooling in an Al-30wt\% Cu alloy, Acta Mater. 53 (2005) 947-956.

[25] R.H. Mathiesen, L. Arnberg, P. Bleuet, A. Somogyi, Crystal fragmentation and columnar-to-equiaxed transitions in Al-Cu studied by synchrotron X-ray video microscopy, Metall. Mater. Trans. A 37 (2006) 2515-2524.

[26] A.J. Clarke, D. Tourret, S.D. Imhoff, P.J. Gibbs, K. Fezzaa, J.C. Cooley, W.-K. Lee, A. Deriy, B.M. Patterson, P.A. Papin, K.D. Clarke, R.D. Field, J.L. Smith, X-ray Imaging and Controlled Solidification of Al-Cu Alloys Toward Microstructures by Design, Adv. Eng. Mat. 17 (2015) 454-459.

[27] J.D. Hunt, S. Lu, Numerical modeling of cellular/dendritic array growth: spacing and structure predictions, Metall. Mater. Trans. A 27 (1996) 611-623.

[28] W. Kurz, D.J. Fisher, Fundamentals of solidification, 3rd ed., Trans Tech Pub, Aedermannsdorf, 1992.

[29] W.W. Mullins, R.F. Sekerka, Stability of a Planar Interface During Solidification of a Dilute Binary Alloy, J. Appl. Phys. 35 (1964) 444-451.

[30] W.A. Tiller, K.A. Jackson, J.W. Rutter, B. Chalmers, The redistribution of solute atoms during the solidification of metals, Acta Metall. 1 (1953) 428-437..

[31] M. Hennenberg, B. Billia, J. Physique 1 (1991) 79-95..

[32] A. Barbieri, J.S. Langer, Predictions of dendritic growth rates in the linearized solvability theory, Phys. Rev. A 39 (1989) 5314. 
[33] M. Ben Amar, E. Brener, Theory of Pattern Selection in Three-Dimensional Nonaxisymmetric Dendritic Growth, Phys. Rev. Lett. 71 (1993) 589.

[34] S. Gurevich, A. Karma, M. Plapp, R. Trivedi, Phase-field study of three-dimensional steady-state growth shapes in directional solidification, Phys. Rev. E 81 (2010) 011603.

[35] S. Lu, J.D. Hunt, A numerical analysis of dendritic and cellular array growth: the spacing adjustment mechanisms, J. Cryst. Growth 123 (1992) 17-34.

[36] J.A. Warren, J.S. Langer, Prediction of dendritic spacings in a directional-solidification experiment, Phys. Rev. E 47 (1993) 2702.

[37] H. Weidong, G. Xingguo, Z. Yaohe, Primary spacing selection of constrained dendritic growth, J. Cryst. Growth 134 (1993) 105-115.

[38] M. Georgelin, A. Pocheau, Oscillatory instability, limit cycle, and transition to doublets in directional solidification, Phys. Rev. Lett. 79 (1997) 2698.

[39] M. Plapp, Three-dimensional phase-field simulations of directional solidification, J. Cryst. Growth 303 (2007) 49-57.

[40] N. Bergeon, D. Tourret, L. Chen, J.-M. Debierre, R. Guérin, A. Ramirez, B. Billia, A. Karma, R. Trivedi, Spatiotemporal dynamics of oscillatory cellular patterns in three-dimensional directional solidification, Phys. Rev. Lett. 110 (2013) 226102.

[41] D. Tourret, J.-M. Debierre, Y. Song, F.L. Mota, N. Bergeon, R. Guérin, R. Trivedi, B. Billia, A. Karma, Oscillatory cellular patterns in three-dimensional directional solidification, Phys. Rev. E 92 (2015) 042401.

[42] K. Kassner, C. Misbah, R. Baumann, Eutectic dynamics: A host of new states, Phys. Rev. E 51 (1995) R2751.

[43] M. Apel, B. Böttger, H.-J. Diepers, I. Steinbach, 2D and 3D phase-field simulations of lamella and fibrous eutectic growth, J. Cryst. Growth 237 (2002) 154-158.

[44] I. Steinbach, Effect of interface anisotropy on spacing selection in constrained dendrite growth, Acta Mater. 56 (2008) 4965-4971.

[45] B. Echebarria, A. Karma, S. Gurevich, Onset of sidebranching in directional solidification, Phys. Rev. E 81 (2010) 021608.

[46] D. Ma, Modeling of Primary Spacing Selection in Dendrite Arrays during Directional Solidification, Metall. Mater. Trans. B 33 (2002) 223-233..

[47] D. Tourret, A. Karma, Multiscale dendritic needle network model of alloy solidification, Acta Mater. 17 (2013) $6474-6491$.

[48] D. Tourret, A.J. Clarke, S.D. Imhoff, P.J. Gibbs, J.W. Gibbs, A. Karma, Three-dimensional multiscale modeling of dendritic spacing selection during Al-Si directional solidification, JOM 67 (2015) 1776-1785.

[49] M.A. Eshelman, V. Seetharaman, R. Trivedi, Cellular spacings — I. Steady-state growth, Acta Metall 36 (1988) $1165-1174$.

[50] V. Seetharaman, M.A. Eshelman, R. Trivedi, Cellular spacings - II. Dynamical studies, Acta Metall 36 (1988) 1175-1185.

[51] K. Miyata, T. Suzuki, J.I. Uno, Cellular and dendritic growth: Part I. Experiment, Metall. Trans. A 16 (1985) $1799-1805$.

[52] G. Grange, J. Gastaldi, C. Jourdan, B. Billia, Evolution of characteristic pattern parameters in directional solidification of thin samples of a dilute Al-Cu alloy, J. Cryst. Growth 151 (1995) 192-199.

[53] J.D. Hunt, Evolution of dendritic patterns during alloy solidification: From the initial instability to the steady state, in: Solidification and Casting of Metals, The Metals Society, London; 1979, pp. 3-9.

[54] S.R. Coriell, M.R. Cordes, W.J. Boettinger, R.F. Sekerka, Convective and interfacial instabilities during unidirectional solidification of a binary alloy, J. Cryst. Growth 49 (1980) 13-28.

[55] H. Nguyen-Thi, B. Billia, H. Jamgotchian, Influence of thermosolutal convection on the solidification front during upwards solidification, J. Fluid. Mech. 204 (1989) 581-597.

[56] H. Jamgotchian, N. Bergeon, D. Benielli, P. Voge, B. Billia, R. Guérin, Localized microstructures induced by fluid flow in directional solidification, Phys. Rev. Lett. 87 (2001) 166105.

[57] N. Shevchenko, S. Boden, G. Gerbeth, S. Eckert, Chimney formation in solidifying Ga-25wt pct In alloys under the influence of thermosolutal melt convection, Metall. Mater. Trans. A 44 (2013) 3797-3808. 
[58] J.W. Gibbs, D. Tourret, P.J. Gibbs, S.D. Imhoff, M.J. Gibbs, B. Walker, K. Fezzaa, A.J. Clarke, In Situ X-Ray Observations of Dendritic Fragmentation During Directional Solidification of a Sn-Bi Alloy, JOM 68 (2016) $170-177$.

[59] M.D. Dupouy, D. Camel, J.J. Favier, Natural convection in directional dendritic solidification of metallic alloys - I. Macroscopic effects, Acta Metall. 37 (1989) 1143-1157.

[60] R. Trivedi, P. Mazumder, S.N. Tewari, The effect of convection on disorder in primary cellular and dendritic arrays, Metall. Mater. Trans. A 33 (2002) 3763-3775.

[61] I. Steinbach, Pattern formation in constrained dendritic growth with solutal buoyancy, Acta Mater. 57 (2009) $2640-2645$.

[62] A. Bogno, G. Reinhart, A. Buffet, H. Nguyen-Thi, B. Billia, T. Schenk, N. Mangelinck-Noël, N. Bergeon, J. Baruchel, In situ analysis of the influence of convection during the initial transient of planar solidification, J. Cryst. Growth 318 (2011) 1134-1138.

[63] A. Bogno, H. Nguyen-Thi, A. Buffet, G. Reinhart, B. Billia, N. Mangelinck-Noël, N. Bergeon, J. Baruchel, T. Schenk, Analysis by synchrotron X-ray radiography of convection effects on the dynamic evolution of the solid-liquid interface and on solute distribution during the initial transient of solidification, Acta Mater. 59 (2011) 4356-4365.

[64] H. Nguyen-Thi, A. Bogno, G. Reinhart, B. Billia, R.H. Mathiesen, G. Zimmermann, Y. Houltz, K. Löth, D. Voss, A. Verga, F. de Pascale, Investigation of gravity effects on solidification of binary alloys with in situ X-ray radiography on earth and in microgravity environment, J. Phys.: Conf. Ser. 327 (2011) 012012.

[65] J.-H. Jeong, N. Goldenfeld, J. Dantzig, Phase field model for three-dimensional dendritic growth with fluid flow, Phys. Rev. E 64 (2001) 041602.

[66] Q. Li, C. Beckermann, Modeling of free dendritic growth of succinonitrile-acetone alloys with thermosolutal melt convection, J. Cryst. Growth 236 (2002) 482-498.

[67] J.-H. Jeong, J. Dantzig, N. Goldenfeld, Dendritic growth with fluid flow in pure materials, Metall. Mater. Trans. A 34 (2003) 459-466.

[68] Y. Lu, C. Beckermann, J.C. Ramirez, Three-dimensional phase-field simulations of the effect of convection on free dendritic growth, J. Cryst. Growth 280 (2005) 320-334.

[69] D. Medvedev, T. Fischaleck, K. Kassner, Influence of external flows on crystal growth: Numerical investigation, Phys. Rev. E 74 (2006) 031606.

[70] D. Medvedev, T. Fischaleck, K. Kassner, Influence of external flows on pattern growth, J. Cryst. Growth 303 (2007) 69-73.

[71] S. Liu, J. Li, J. Lee, R. Trivedi, Spatio-temporal microstructure evolution in directional solidification processes, Phil. Mag. 86 (2006) 3717-3738.

[72] H.-J. Diepers, I. Steinbach, Interaction of interdendritic convection and dendritic primary spacing: phase-field simulation and analytical modeling, Mater. Sci. Forum 508 (2006) 145-150.

[73] B. Echebarria, R. Folch, A. Karma, M. Plapp, Quantitative phase-field model of alloy solidification, Phys. Rev. E 70 (2004) 061604.

[74] D. Tourret, A. Karma, Growth competition of columnar dendritic grains: A phase-field study, Acta Mater. 82 (2015) 64-83.

[75] A. Karma, Phase-Field Formulation for Quantitative Modeling of Alloy Solidification, Phys. Rev. Lett. 87 (2001) 115701.

[76] A. Karma, W.J. Rappel, Quantitative phase-field modeling of dendritic growth in two and three dimensions, Phys. Rev. E 57 (1998) 4323.

[77] K. Glasner, Nonlinear Preconditioning for Diffuse Interfaces, J. Comput. Phys. 174 (2001) 695-711.

[78] A. Karma, W.J. Rappel, Phase-field model of dendritic sidebranching with thermal noise, Phys. Rev. E 60 (1999) 3614.

[79] J.A. Warren, T. Pusztai, L. Környei, L. Gránásy, Phase field approach to heterogeneous crystal nucleation in alloys, Phys. Rev. B 79 (2009) 014204.

[80] J. Ghmadh, J.-M. Debierre, J. Deschamps, M. Georgelin, R. Guérin, A. Pocheau, Directional solidification of inclined 
structures in thin-samples, Acta Mater. 74 (2014) 255-267.

[81] C. Beckermann, H.-J. Diepers, I. Steinbach, A. Karma, X. Tong, Modeling melt convection in phase-field simulations of solidification, J. Comput. Phys. 154 (1999) 468-496.

[82] J.P. Garandet, T. Duffar, J.J. Favier, On the scaling analysis of the solute boundary layer in idealized growth configurations, J. Cryst. Growth 106 (1990) 437-444.

[83] J.P. Garandet, J.J. Favier, D. Camel, Solutal boundary layer concept and scaling analysis: two keys to segregation phenomena in melt crystal growth, J. Cryst. Growth 130 (1993) 113-122.

[84] J.A. Burton, R.C. Prim, W.P. Slichter, The distribution of solute in crystals grown from the melt. Part I. Theoretical, J. Chem. Phys. 21 (1953) 1987-1991.

[85] B. Cantor, A. Vogel, Stability of a spherical particle growing from a stirred melt, J. Cryst. Growth 37 (1977) $309-316$.

[86] D.T.J. Hurle, Interface stability during the solidification of a stirred binary-alloy melt, J. Cryst. Growth 5 (1969) $162-166$.

[87] S.R. Coriell, D.T.J. Hurle, R.F. Sekerka, Interface stability during crystal growth: the effect of stirring, J. Cryst. Growth 32 (1976) 1-7.

[88] B. Cantor, A. Vogel, Dendritic solidification and fluid flow, J. Cryst. Growth 41 (1977) $109-123$.

[89] R.F. Sekerka, S.R. Coriell, G.B. McFadden, Stagnant film model of the effect of natural convection on the dendrite operating state, J. Cryst. Growth 154 (1995) 370-376.

[90] D.S. Schrage, A simplified model of dendritic growth in the presence of natural convection, J. Cryst. Growth 205 (1999) 410-426.

[91] Ch.-A. Gandin, G. Guillemot, B. Appolaire, N.T. Niane, Boundary layer correlation for dendrite tip growth with fluid flow, Mater. Sci. Eng. A 342 (2003) 44-50.

[92] D. Camel, J.J. Favier, Scaling analysis of convective solute transport and segregation in Bridgman crystal growth from the doped melt, J. Phys. 47 (1986) 1001-1014.

[93] B. Drevet, J.P. Garandet, D. Camel, J.J. Favier, Influence of thermal bouyancy on fibrous eutectic growth I. Segregation results in the Sn-Cu6Sn5 system, J. Cryst. Growth 129 (1993) 549-558.

[94] A. Karma, W.J. Rappel, B.C. Fuh, R. Trivedi, Model of banding in diffusive and convective regimes during directional solidification of peritectic systems, Metall. Mater. Trans. A 29 (1998) 1457-1470.

[95] P. Mazumder, R. Trivedi, A. Karma, A model of convection-induced oscillatory structure formation in peritectic alloys, Metall. Mater. Trans. A 31 (2000) 1233-1246.

[96] J.H. Lee, S. Liu, H. Miyahara, R. Trivedi, Diffusion-coefficient measurements in liquid metallic alloys, Metall. Mater. Trans. B 35 (2004) 909-917.

[97] M. Gündüz, J.D. Hunt, The measurement of solid-liquid surface energies in the Al-Cu, Al-Si and Pb-Sn systems, Acta Metall. 33 (1985) 1651-1672.

[98] J.R. Morris, The Anisotropic Free Energy of the Solid-Liquid Phase Boundary in Al, Phys. Rev. B 66 (2002) 144104.

[99] M. Tassa, J.D. Hunt, The measurement of Al-Cu dendrite tip and eutectic interface temperatures and their use for predicting the extent of the eutectic range, J. Cryst. Growth 34 (1976) 38-48.

[100] J.L. Murray, The Aluminium-Cooper system, Int. Metals Rev. 30 (1985) 211-233.

[101] D. Tourret, A. Karma, A.J. Clarke, P.J. Gibbs, S.D. Imhoff, Three-dimensional Dendritic Needle Network model with application to Al-Cu directional solidification experiments, IOP Conf. Ser.: Mater. Sci. Eng. 84 (2015) 012082.

[102] H.-J. Diepers, D. Ma, I. Steinbach, History effects during the selection of primary dendrite spacing. Comparison of phase-field simulations with experimental observations, J. Cryst. Growth 237 (2002) 149-153.

[103] S. Gurevich, M. Amoorezaei, N. Provatas, Phase-field study of spacing evolution during transient growth, Phys. Rev. E $82(2010) 051606$.

[104] M. Amoorezaei, S. Gurevich, N. Provatas, Spacing characterization in Al-Cu alloys directionally solidified under transient 
growth conditions, Acta Mater. 58 (2010) 6115-6124.

[105] P. Bouissou, P. Pelcé, Effect of a forced flow on dendritic growth, Phys. Rev. A 40 (1989) 6673.

[106] T. Takaki, R. Rojas, M. Ohno, T. Shimokawabe, T. Aoki, GPU phase-field lattice Boltzmann simulations of growth and motion of a binary alloy dendrite, IOP Conf. Ser.: Mater. Sci. Eng. 84 (2015) 012066. 


\section{List of Figures}

1 Schematic microstructure selection map. The gray box shows the typical range of parameters for the Al-1.4wt\% Cu alloy in our experiments (see Fig. 11 in Ref. [27]). . . . . . . . . . . . .

2 Typical solution for a steady $1 \mathrm{D}$ diffuse interface propagating at a velocity $V_{0}$, yielding a phase field profile $\varphi \sim\left(x-V_{0} t\right) / W$, here represented for $W=1$ (dashed line), and corresponding preconditioned phase field $\psi$ (solid line), with $\varphi \equiv \tanh (\psi / \sqrt{2})$. . . . . . . . . . .

3 Schematics of the boundary-layer (BL) approach for mixed diffusive-convective transport. Illustrated here for $G=50 \mathrm{~K} / \mathrm{cm}$ and $V=10 \mu \mathrm{m} / \mathrm{s}$, with $\left(x_{t i p}, y_{t i p}, z_{t i p}\right)$ the coordinates of the tip of a dendrite: (a) shows typical concentration profiles along the $(y, z)=\left(y_{\text {tip }}, z_{\text {tip }}\right)$ line, i.e. the dashed line in (c), for different values of the diffusive BL thickness $\delta$; (b) shows the preconditioned phase field $\psi$ along the $(y, z)=\left(y_{\text {tip }}, z_{\text {tip }}\right)$ line; and (c) illustrates isovalues of $\psi$ in front of a dendritic array on the $\left(z=z_{\text {tip }}\right)$ plane for the case purely diffusive case, i.e.

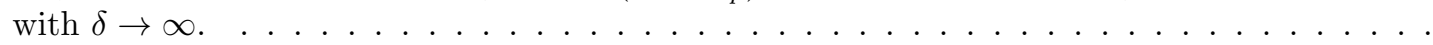

4 Simulated interface pattern after 600 seconds in the spatially extended simulation with $G=$ $50 \mathrm{~K} / \mathrm{cm}, V=20 \mu \mathrm{m} / \mathrm{s}$ and $\delta=100 \mu \mathrm{m}$, exhibiting an inhomogeneous microstructure throughout the domain. The thin black box shows the size of the whole simulation domain, with periodic boundary conditions in the $y$-direction. . . . . . . . . . . . . .

5 Primary tip radii calculation from phase-field results, illustrated for $G=50 \mathrm{~K} / \mathrm{cm}, V=$ $15 \mu \mathrm{m} / \mathrm{s}$ and $\delta \rightarrow \infty$. For each tip position $\left(y_{t i p}, z_{t i p}\right)$, the intersections of the $(\psi=0)$ solidliquid interface with planes $\left(y=y_{t i p}\right)$ and $\left(z=z_{t i p}\right)$, respectively in red and blue in (a), are tabulated as $y_{i}(x)$ and $z_{i}(x)$, in gray lines in (b) and (c). These interface locations are fitted to parabolic functions (color plots) to calculate the radii $\rho_{y}$ (b) and $\rho_{z}$ (c). For the sake of simplicity, the example here has only one row of dendrites such that all $\rho_{y}$ can be represented on a unique $\left(z=z_{\text {tip }}\right)$ plane in (b). The fitted radii $\rho_{y}$ and $\rho_{z}$ for the leftmost dendrite are plotted as a function of the fitting range length in $(\mathrm{d})$, together with the $\left(\rho=\left|x-x_{\text {tip }}\right|\right)$ line. The final $\rho_{y}$ and $\rho_{z}$ for a fitting range spanning one tip radius behind the tip appear in full symbols in $(\mathrm{d}) . \ldots \ldots \ldots \ldots \ldots \ldots \ldots \ldots \ldots$

6 X-ray radiographs of directional solidification experiments at two different temperature gradients $G=50$ (left) and $G=80 \mathrm{~K} / \mathrm{cm}$ (right) for different growth velocities $V$. . . . . . . .

7 Selected microstructures in spatially extended phase-field simulations for $G=50 \mathrm{~K} / \mathrm{cm}$ with $V=5,10,15,20$, and $50 \mu \mathrm{m} / \mathrm{s}$ (rows), for different values of $\delta=\infty, 200$, and $100 \mu \mathrm{m}$ (columns). The thick orange line in the background illustrates the limit between dominant diffusion with $D / V<\delta$ (bottom-left) and dominant convection with $\delta<D / V$ (top-right).

8 Microstructural selection map from directional solidification experiments. The thick black line represents the planar instability limit in the diffusive regime, i.e. Eq. (2). Symbols represent experiments resulting in a planar $(\diamond)$, cellular $(\circ)$, or dendritic $(\triangle)$ microstructure. Open symbols stand for a growth direction from the bottom upward, and larger full symbols in a slightly lighter color correspond to a growth for the top downward. The solid green and dashed red lines illustrate the approximate limits between different growth regimes in the bottom-up experiments. . . . . . . . . . . . . . . . . . . .

9 Planar front stability critical velocity $V_{c}$ as a function of the boundary layer thickness $\delta$ for $G=50 \mathrm{~K} / \mathrm{cm}$ (a). The analytical expression (3) for $V_{c}(\delta)$ (solid red line) is compared to results of phase-field simulations, described in Section 3.3.2, yielding a planar $(\triangle)$ or nonplanar $(\nabla)$ interface. The experimentally assessed value of $V_{c}$ is marked with a horizontal dashed green line. For each simulation, the time evolution of the amplitude of the interface shape $\Delta x_{i}=x_{t i p}-x_{\text {groove }}$ (see Sec. 3.3.2) appears in (b), with stable planar interfaces in dashed lines and unstable interfaces in solid lines. . . . . . . . . . . . .

10 Average primary spacings measured by in situ imaging (symbol) with spacing distribution range (uncertainty limits) for different directional solidification experiments. . . . . . . . . . 35 
11 Time evolution toward a homogeneous microstructure after planar interface destabilization, for $G=50 \mathrm{~K} / \mathrm{cm}, V=20 \mu \mathrm{m} / \mathrm{s}$ and $\delta=100 \mu \mathrm{m}$. The evolution of the average $(\Lambda)$, minimum $\left(\Lambda_{\text {low }}\right)$, maximum $\left(\Lambda_{\text {high }}\right)$ spacings, as well as the average spacing in the one-row $\left(\Lambda_{\text {lr }}\right)$ and two-row $\left(\Lambda_{2 \mathrm{r}}\right)$ regions, as defined in Fig. 4b, appears in (a). Snapshots (b)-(i) show the evolution of the pattern from a two-row (red) to a one-row (blue) microstructure by slow progressive elimination of dendrites. The movie of the evolution from (b) to (i) is attached as Supplementary Material. . . . . . . . . . . . . . . . . . . . . .

12 Primary spacings in spatially extended simulations in the one-row (open symbols) and tworow (full symbols) regions versus growth velocity $V$ for different BL thicknesses $\delta$, compared to experimental measurements for $G=50 \mathrm{~K} / \mathrm{cm}(\times)$. Shaded backgrounds behind each curve represents the scatter of all spacings measured within the sample, i.e. the region between $\Lambda_{\text {low }}$ and $\Lambda_{\text {high }}$ (see Fig. 4). The gray dotted line shows a fit to $\Lambda \sim V^{-1 / 2}$ for $\delta \rightarrow \infty$. . .

13 Stability limits of primary spacing $\Lambda_{\max }$ (a) and $\Lambda_{\min }$ (b) versus growth velocity $V$ for different BL thicknesses $\delta$, compared to experimental measurements for $G=50 \mathrm{~K} / \mathrm{cm}(\times)$. The fits to $\Lambda \sim V^{-1 / 2}$ for $\Lambda_{\min }$ and $\Lambda_{\max }$ with $\delta \rightarrow \infty$ appear as gray dotted lines. . . . . . . . . 38

14 Average primary tip radii measured by in situ X-ray imaging (symbol) with spacing distribution range (uncertainty limits) for different directional solidification experiments. . . . . . .

15 Average primary tip radii $\rho(\mathrm{a}), \rho_{y}(\mathrm{~b}), \rho_{z}(\mathrm{c})$ measured in spatially extended simulations in the one-row (open symbols) and two-row (full symbols) regions versus growth velocity $V$ for different BL thicknesses $\delta$. Experimental measurements for $G=50 \mathrm{~K} / \mathrm{cm}$ appear in black $\times$ symbols for direct comparison with $\rho_{y}$ in (b) and in gray symbols in (a) and (c). The gray dotted line in (a)-(b) shows the fit $\rho \sim V^{-1 / 2}$ for all values of $\delta \ldots \ldots \ldots \ldots . . \ldots$ 


\section{Figures}

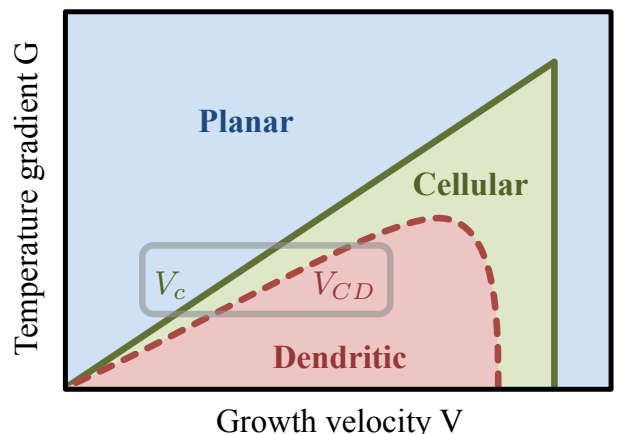

Figure 1: Schematic microstructure selection map. The gray box shows the typical range of parameters for the $\mathrm{Al}-1.4 \mathrm{wt} \% \mathrm{Cu}$ alloy in our experiments (see Fig. 11 in Ref. [27]). 


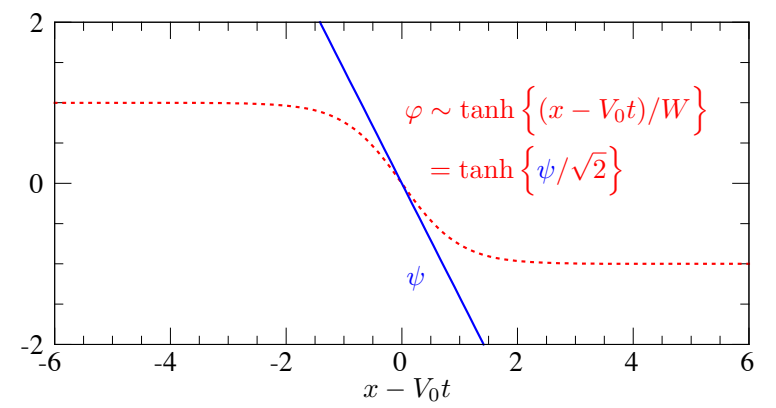

Figure 2: Typical solution for a steady 1D diffuse interface propagating at a velocity $V_{0}$, yielding a phase field profile $\varphi \sim$ $\left(x-V_{0} t\right) / W$, here represented for $W=1$ (dashed line), and corresponding preconditioned phase field $\psi$ (solid line), with $\varphi \equiv \tanh (\psi / \sqrt{2})$. 

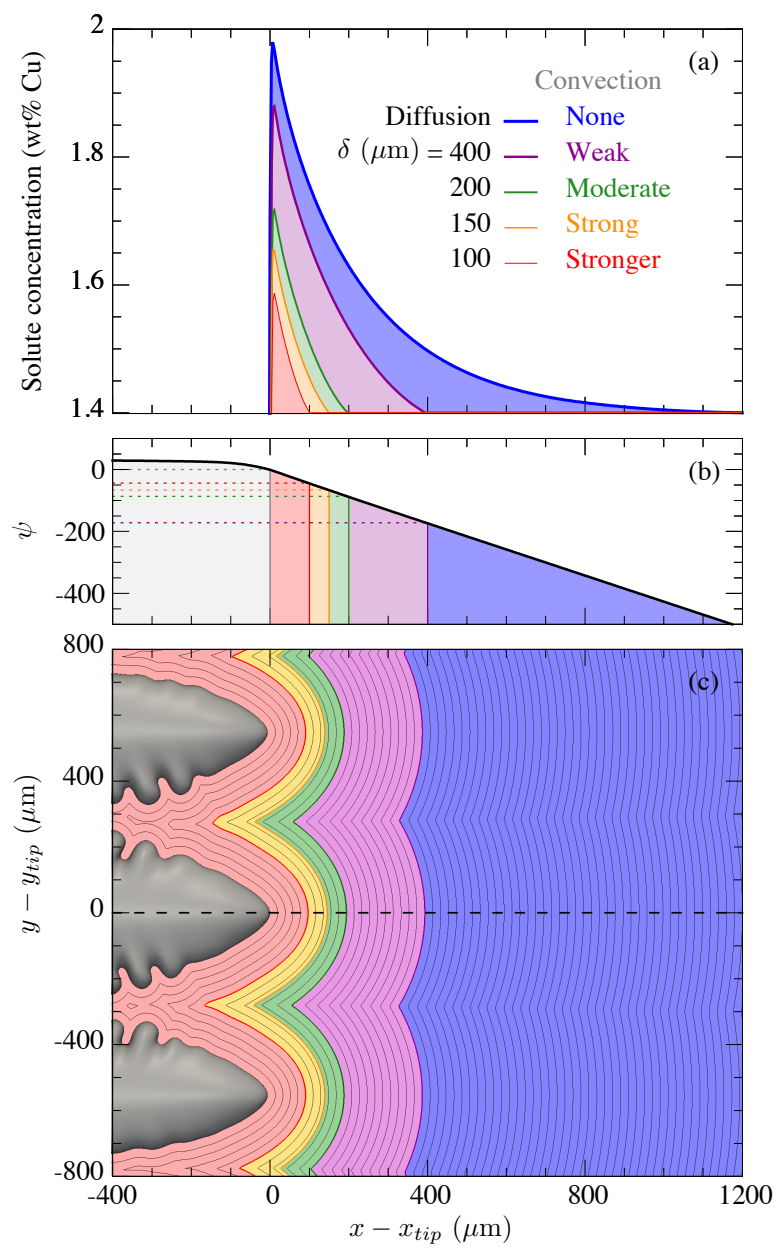

Figure 3: Schematics of the boundary-layer (BL) approach for mixed diffusive-convective transport. Illustrated here for $G=50 \mathrm{~K} / \mathrm{cm}$ and $V=10 \mu \mathrm{m} / \mathrm{s}$, with $\left(x_{t i p}, y_{t i p}, z_{t i p}\right)$ the coordinates of the tip of a dendrite: (a) shows typical concentration profiles along the $(y, z)=\left(y_{t i p}, z_{t i p}\right)$ line, i.e. the dashed line in (c), for different values of the diffusive BL thickness $\delta$; (b) shows the preconditioned phase field $\psi$ along the $(y, z)=\left(y_{\text {tip }}, z_{\text {tip }}\right)$ line; and (c) illustrates isovalues of $\psi$ in front of a dendritic array on the $\left(z=z_{t i p}\right)$ plane for the case purely diffusive case, i.e. with $\delta \rightarrow \infty$. 
(a)

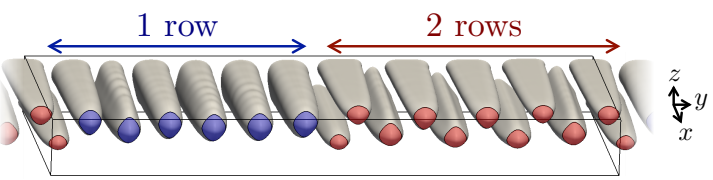

(b)

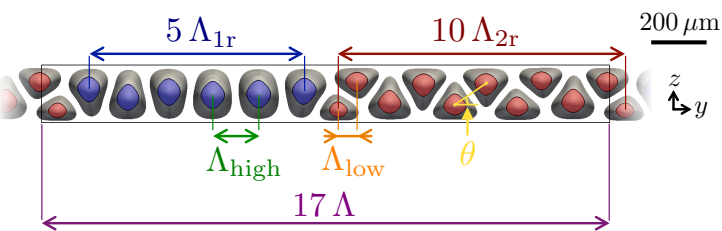

Figure 4: Simulated interface pattern after 600 seconds in the spatially extended simulation with $G=50 \mathrm{~K} / \mathrm{cm}, V=20 \mu \mathrm{m} / \mathrm{s}$ and $\delta=100 \mu \mathrm{m}$, exhibiting an inhomogeneous microstructure throughout the domain. The thin black box shows the size of the whole simulation domain, with periodic boundary conditions in the $y$-direction. 

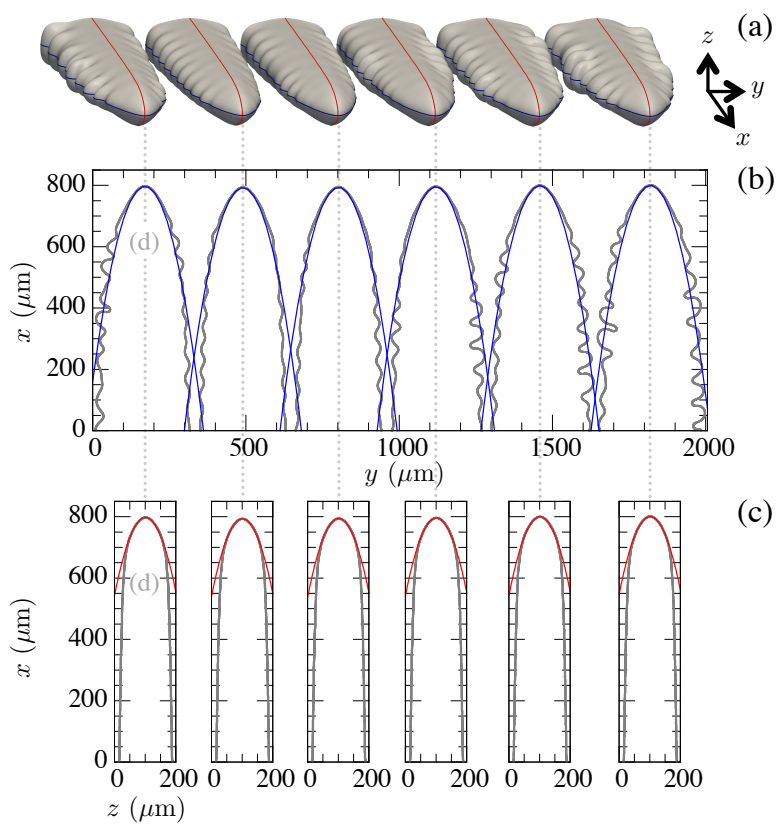

(c)

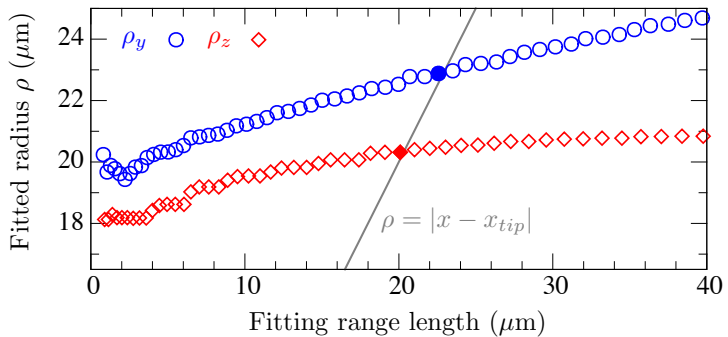

(d)

Figure 5: Primary tip radii calculation from phase-field results, illustrated for $G=50 \mathrm{~K} / \mathrm{cm}, V=15 \mu \mathrm{m} / \mathrm{s}$ and $\delta \rightarrow \infty$. For each tip position $\left(y_{t i p}, z_{t i p}\right)$, the intersections of the $(\psi=0)$ solid-liquid interface with planes $\left(y=y_{\text {tip }}\right)$ and $\left(z=z_{\text {tip }}\right)$, respectively in red and blue in (a), are tabulated as $y_{i}(x)$ and $z_{i}(x)$, in gray lines in (b) and (c). These interface locations are fitted to parabolic functions (color plots) to calculate the radii $\rho_{y}$ (b) and $\rho_{z}$ (c). For the sake of simplicity, the example here has only one row of dendrites such that all $\rho_{y}$ can be represented on a unique $\left(z=z_{\text {tip }}\right)$ plane in (b). The fitted radii $\rho_{y}$ and $\rho_{z}$ for the leftmost dendrite are plotted as a function of the fitting range length in (d), together with the $\left(\rho=\left|x-x_{t i p}\right|\right)$ line. The final $\rho_{y}$ and $\rho_{z}$ for a fitting range spanning one tip radius behind the tip appear in full symbols in (d). 

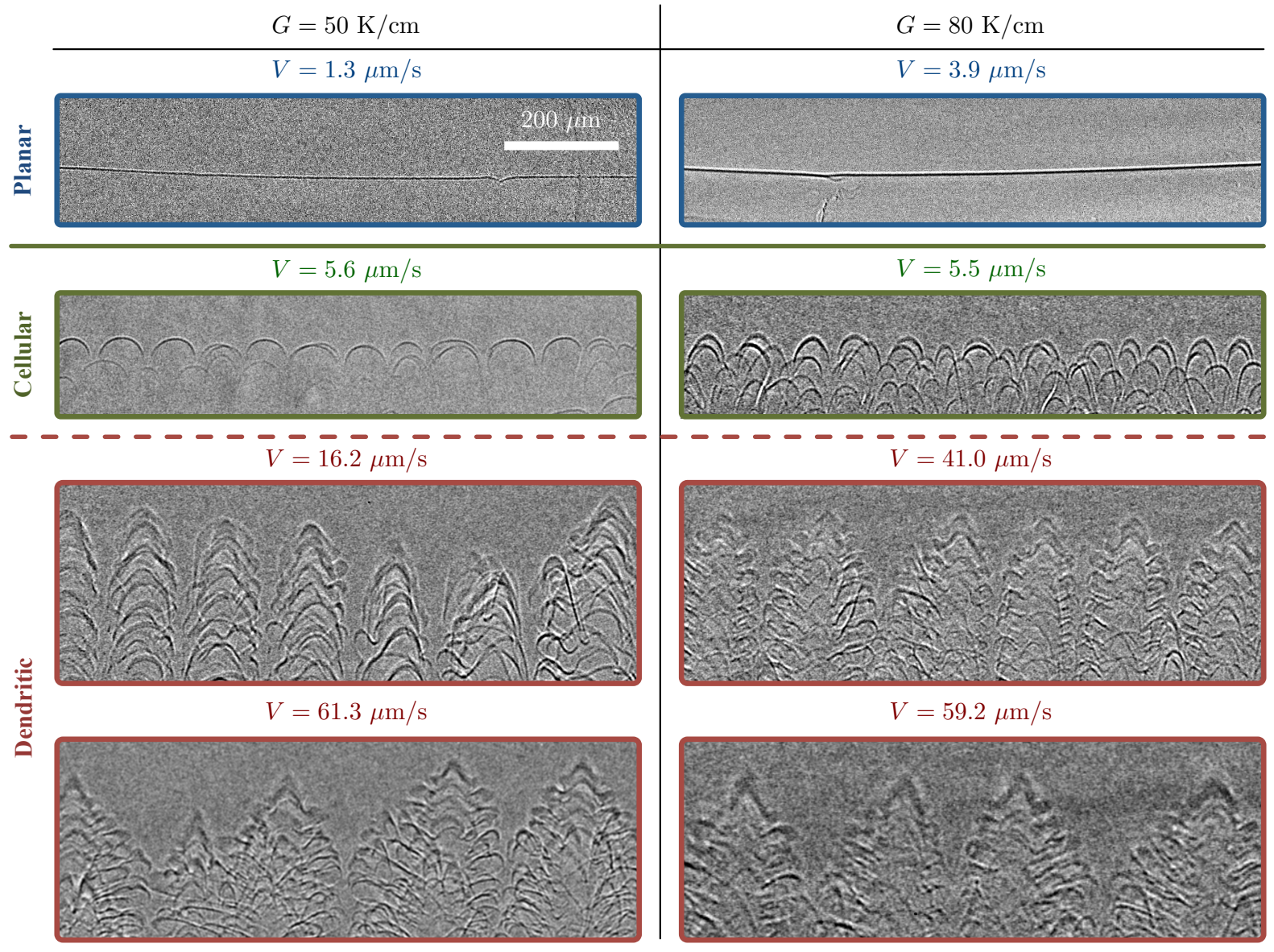

Figure 6: X-ray radiographs of directional solidification experiments at two different temperature gradients $G=50$ (left) and $G=80 \mathrm{~K} / \mathrm{cm}$ (right) for different growth velocities $V$. 


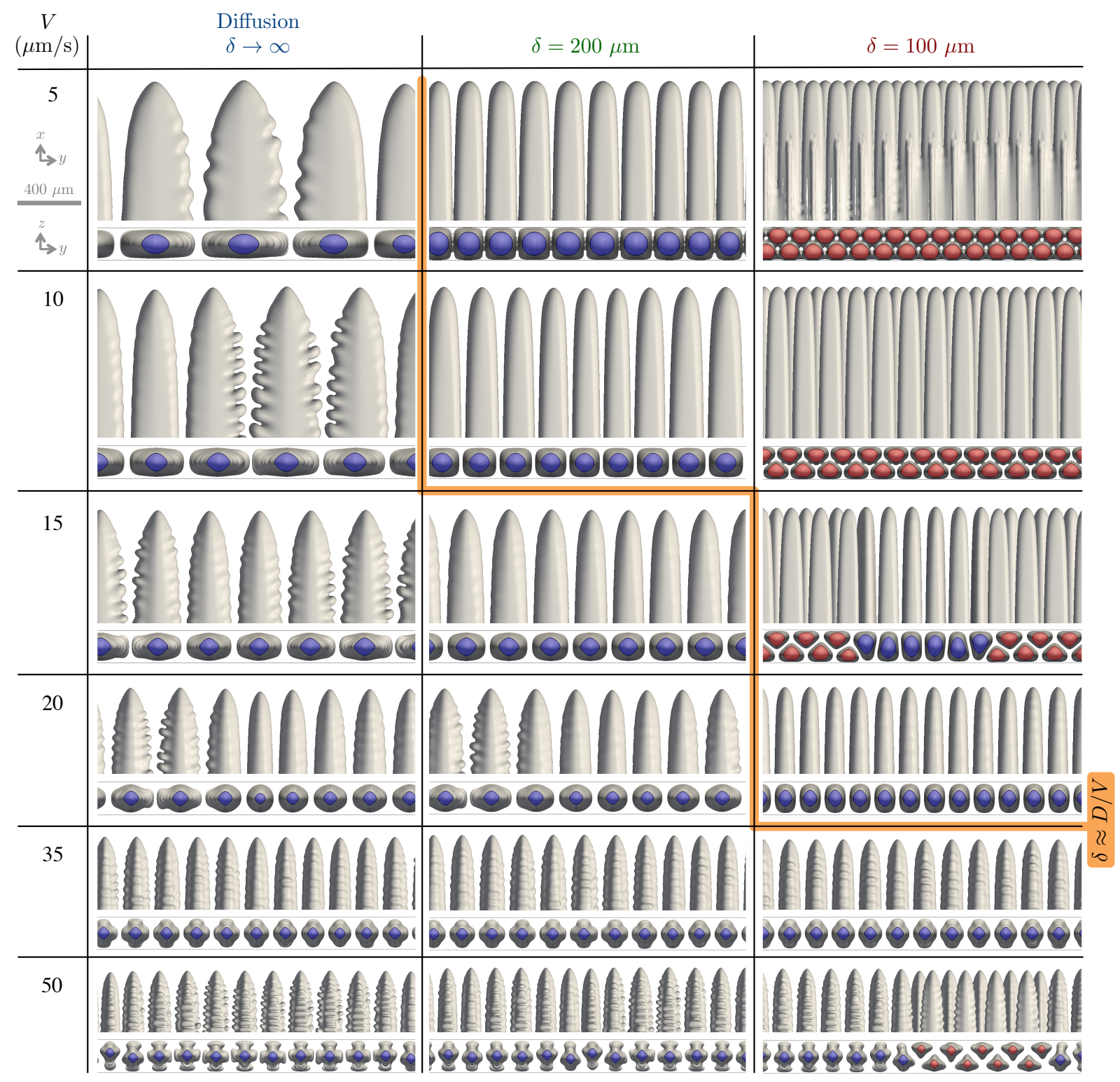

Figure 7: Selected microstructures in spatially extended phase-field simulations for $G=50 \mathrm{~K} / \mathrm{cm}$ with $V=5,10,15,20$, and $50 \mu \mathrm{m} / \mathrm{s}$ (rows), for different values of $\delta=\infty, 200$, and $100 \mu \mathrm{m}$ (columns). The thick orange line in the background illustrates the limit between dominant diffusion with $D / V<\delta$ (bottom-left) and dominant convection with $\delta<D / V$ (top-right). 


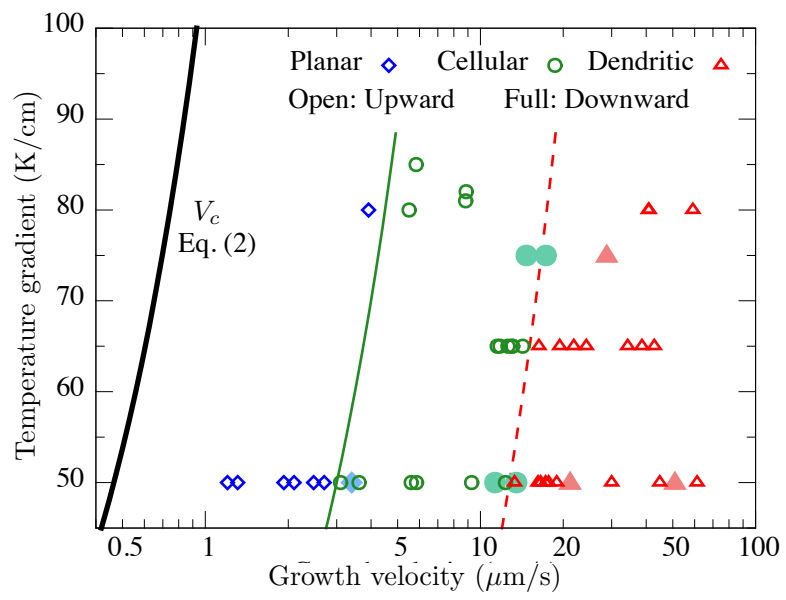

Figure 8: Microstructural selection map from directional solidification experiments. The thick black line represents the planar instability limit in the diffusive regime, i.e. Eq. (2). Symbols represent experiments resulting in a planar $(\diamond)$, cellular $(\circ)$, or dendritic $(\triangle)$ microstructure. Open symbols stand for a growth direction from the bottom upward, and larger full symbols in a slightly lighter color correspond to a growth for the top downward. The solid green and dashed red lines illustrate the approximate limits between different growth regimes in the bottom-up experiments. 

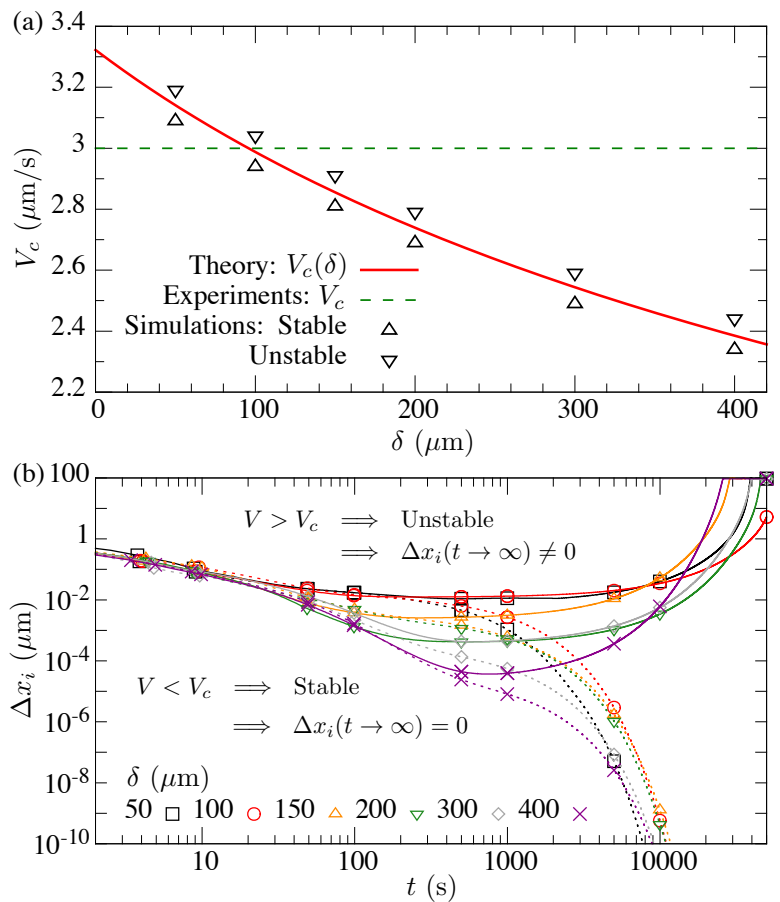

Figure 9: Planar front stability critical velocity $V_{c}$ as a function of the boundary layer thickness $\delta$ for $G=50 \mathrm{~K} / \mathrm{cm}(\mathrm{a})$. The analytical expression $(3)$ for $V_{c}(\delta)$ (solid red line) is compared to results of phase-field simulations, described in Section 3.3.2, yielding a planar $(\triangle)$ or non-planar $(\nabla)$ interface. The experimentally assessed value of $V_{c}$ is marked with a horizontal dashed green line. For each simulation, the time evolution of the amplitude of the interface shape $\Delta x_{i}=x_{\text {tip }}-x_{\text {groove }}$ (see Sec. 3.3.2) appears in (b), with stable planar interfaces in dashed lines and unstable interfaces in solid lines. 


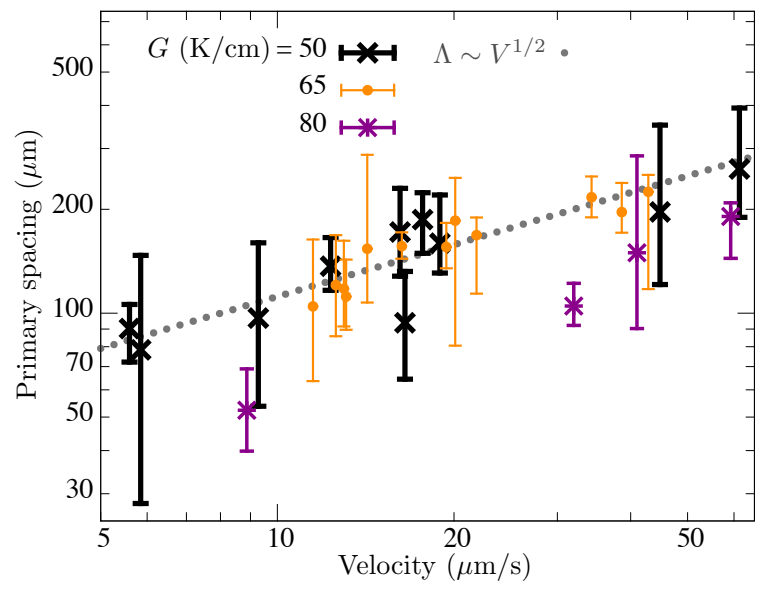

Figure 10: Average primary spacings measured by in situ imaging (symbol) with spacing distribution range (uncertainty limits) for different directional solidification experiments. 

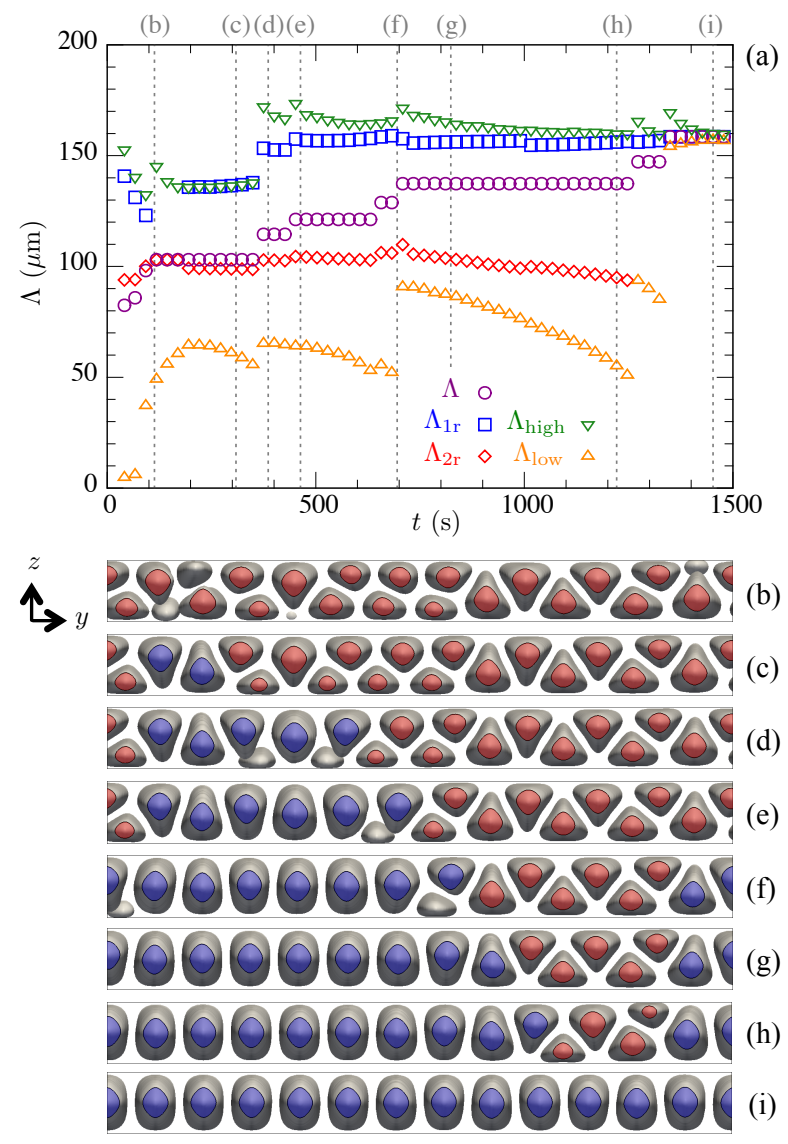

Figure 11: Time evolution toward a homogeneous microstructure after planar interface destabilization, for $G=50 \mathrm{~K} / \mathrm{cm}$, $V=20 \mu \mathrm{m} / \mathrm{s}$ and $\delta=100 \mu \mathrm{m}$. The evolution of the average $(\Lambda)$, minimum $\left(\Lambda_{\text {low }}\right)$, maximum $\left(\Lambda_{\text {high }}\right)$ spacings, as well as the average spacing in the one-row $\left(\Lambda_{1 \mathrm{r}}\right)$ and two-row $\left(\Lambda_{2 \mathrm{r}}\right)$ regions, as defined in Fig. $4 \mathrm{~b}$, appears in (a). Snapshots (b)-(i) show the evolution of the pattern from a two-row (red) to a one-row (blue) microstructure by slow progressive elimination of dendrites. The movie of the evolution from (b) to (i) is attached as Supplementary Material. 


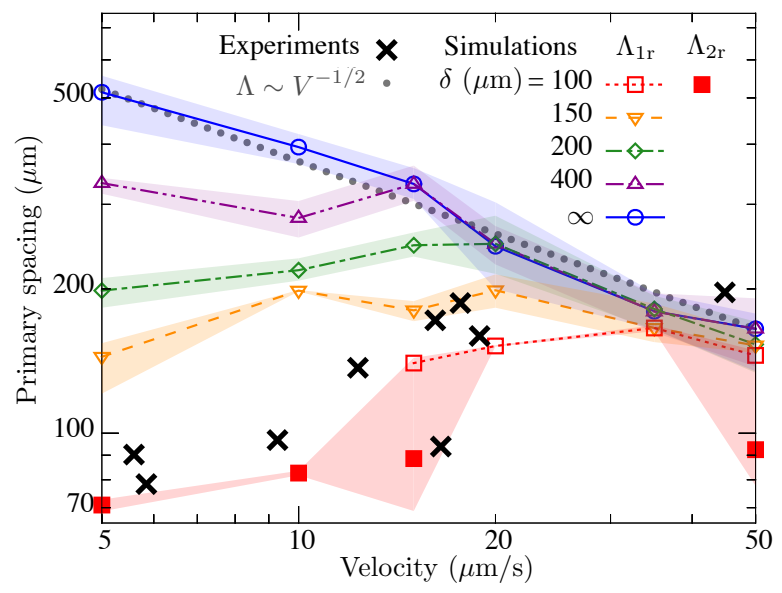

Figure 12: Primary spacings in spatially extended simulations in the one-row (open symbols) and two-row (full symbols) regions versus growth velocity $V$ for different BL thicknesses $\delta$, compared to experimental measurements for $G=50 \mathrm{~K} / \mathrm{cm}(\times)$. Shaded backgrounds behind each curve represents the scatter of all spacings measured within the sample, i.e. the region between $\Lambda_{\text {low }}$ and $\Lambda_{\text {high }}$ (see Fig. 4). The gray dotted line shows a fit to $\Lambda \sim V^{-1 / 2}$ for $\delta \rightarrow \infty$. 


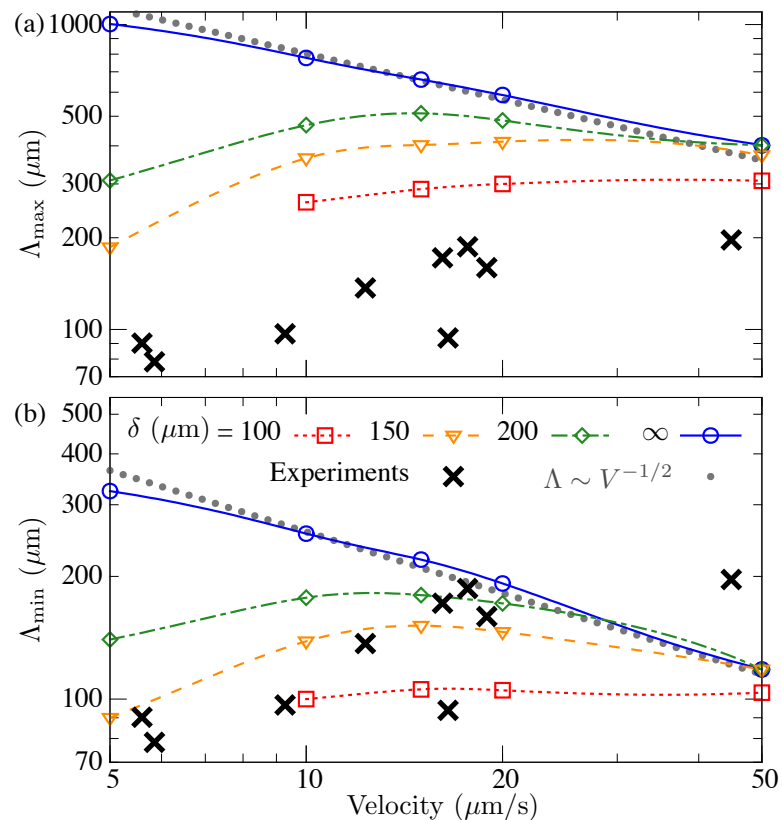

Figure 13: Stability limits of primary spacing $\Lambda_{\max }$ (a) and $\Lambda_{\min }$ (b) versus growth velocity $V$ for different BL thicknesses $\delta$, compared to experimental measurements for $G=50 \mathrm{~K} / \mathrm{cm}(\times)$. The fits to $\Lambda \sim V^{-1 / 2}$ for $\Lambda_{\min }$ and $\Lambda_{\max }$ with $\delta \rightarrow \infty$ appear as gray dotted lines. 


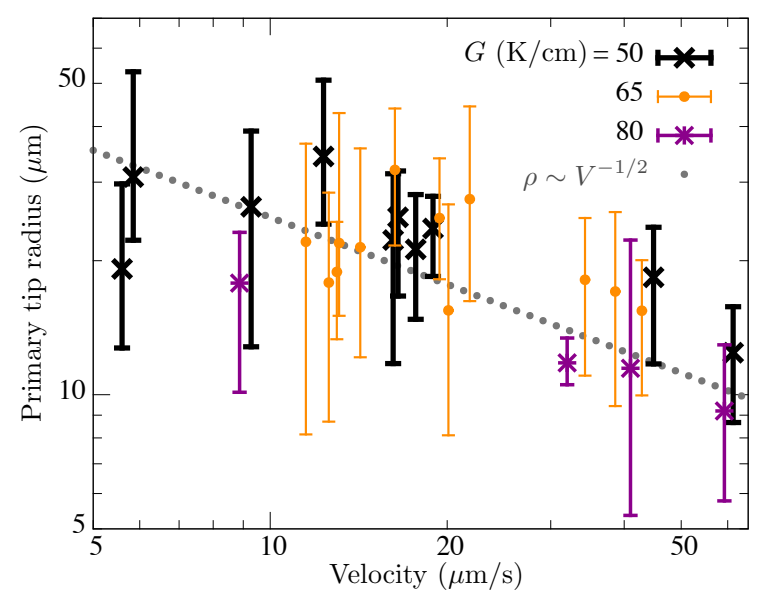

Figure 14: Average primary tip radii measured by in situ X-ray imaging (symbol) with spacing distribution range (uncertainty limits) for different directional solidification experiments. 

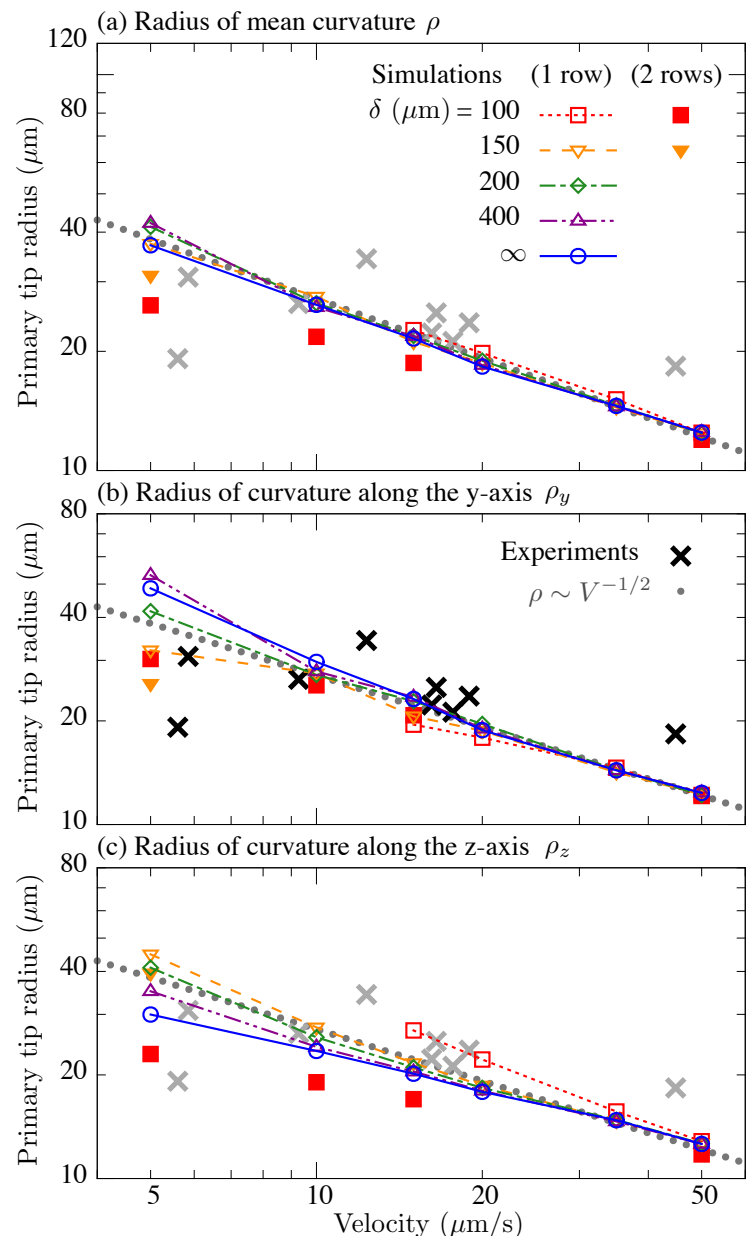

Figure 15: Average primary tip radii $\rho$ (a), $\rho_{y}$ (b), $\rho_{z}$ (c) measured in spatially extended simulations in the one-row (open symbols) and two-row (full symbols) regions versus growth velocity $V$ for different BL thicknesses $\delta$. Experimental measurements for $G=50 \mathrm{~K} / \mathrm{cm}$ appear in black $\times$ symbols for direct comparison with $\rho_{y}$ in (b) and in gray symbols in (a) and (c). The gray dotted line in (a)-(b) shows the fit $\rho \sim V^{-1 / 2}$ for all values of $\delta$ 


\section{List of Tables}

1 Materials and process parameters used in phase-field simulations. For detailed numerical parameters, see the attached Supplementary Material. . . . . . . . . . . . . . . . . . . . . 42

2 Physical simulated time (in minutes) . . . . . . . . . . . . . . . . . . . . 43 


\section{Tables}

Table 1: Materials and process parameters used in phase-field simulations. For detailed numerical parameters, see the attached Supplementary Material.

\begin{tabular}{lrll}
\hline Symbol & Value & Unit & Ref. \\
\hline$c_{\infty}$ & 1.4 & $\mathrm{wt} \% \mathrm{Cu}$ & \\
$D$ & $2.410^{-9}$ & $\mathrm{~m}^{2} / \mathrm{s}$ & {$[96]$} \\
$k$ & 0.14 & & {$[28]$} \\
$m$ & -3.0 & $\mathrm{~K} / \mathrm{wt} \% \mathrm{Cu}$ & (cf. text) \\
$\Gamma$ & $2.410^{-7}$ & $\mathrm{Km}$ & {$[28,97]$} \\
$\epsilon_{4}$ & 0.012 & & {$[98]$} \\
$G$ & 50 & $\mathrm{~K} / \mathrm{cm}$ & \\
$V$ & $5,10,15$, & $\mu \mathrm{m} / \mathrm{s}$ & \\
& $20,35,50$ & & \\
\hline
\end{tabular}


Table 2: Physical simulated time (in minutes).

\begin{tabular}{c|ccccc}
\hline$\delta(\mu \mathrm{m})$ & 100 & 150 & 200 & 400 & $\infty$ \\
\hline$V(\mu \mathrm{m} / \mathrm{s})$ & & & & & \\
5 & 60 & 60 & 34 & 34 & 34 \\
10 & 40 & 30 & 30 & 30 & 20 \\
15 & 26 & 20 & 15 & 12 & 10 \\
20 & 18 & 9 & 9 & 9 & 7 \\
35 & 15 & 9 & 8 & 8 & 7 \\
50 & 15 & 7 & 7 & 6 & 6 \\
\hline
\end{tabular}


Microstructure selection map

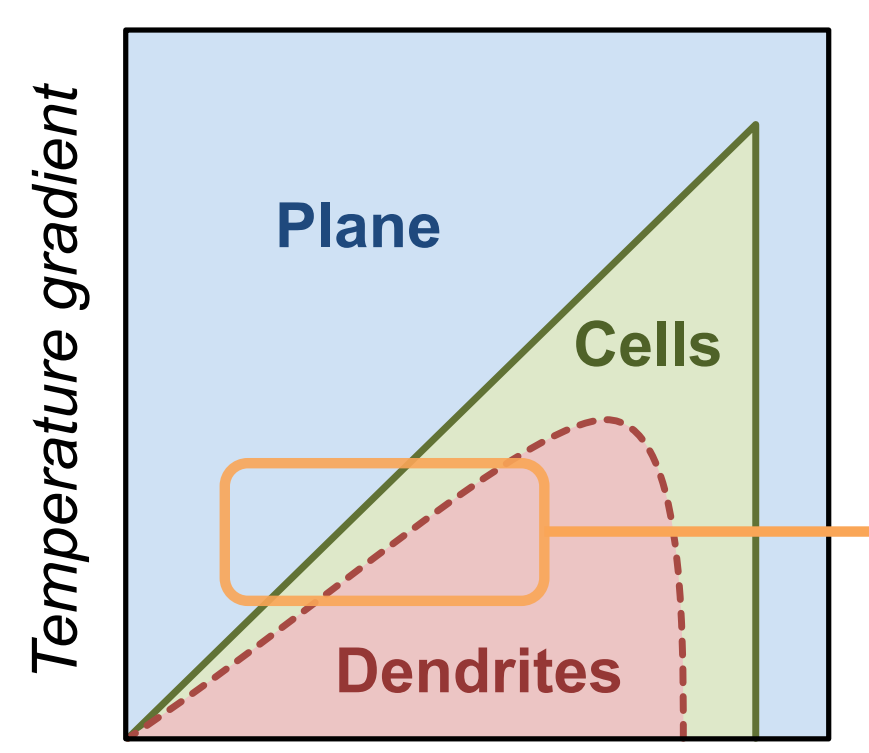

Growth velocity
X-ray imaging of directional solidification experiments

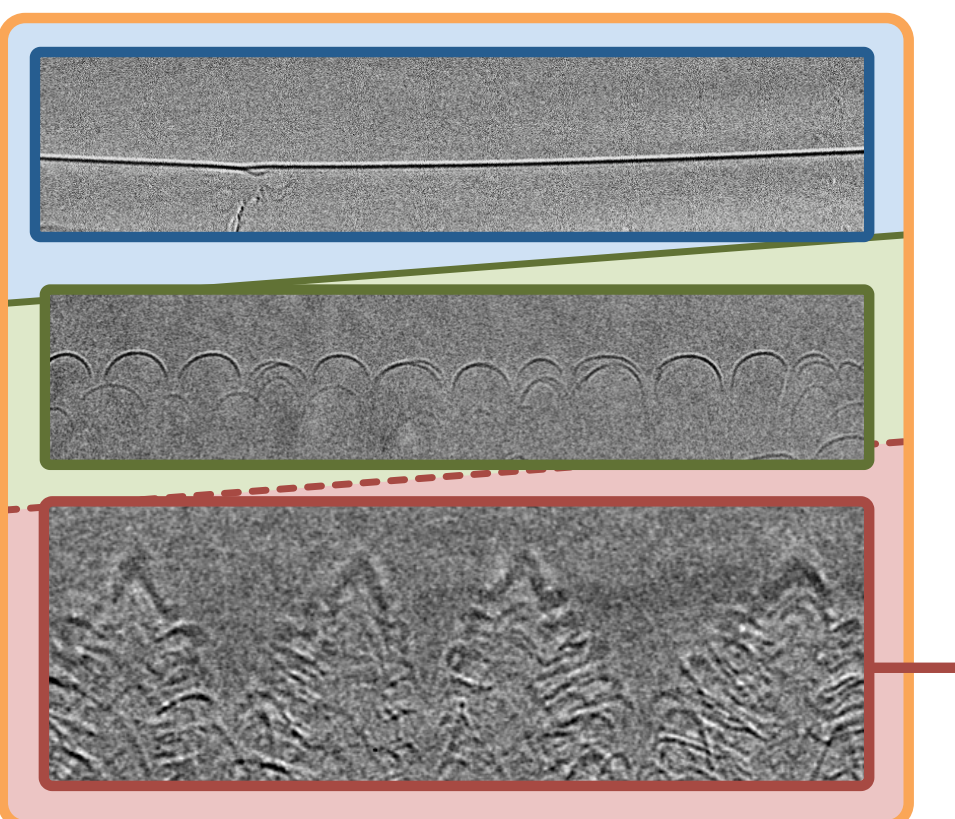

Three-dimensional phase-field simulations

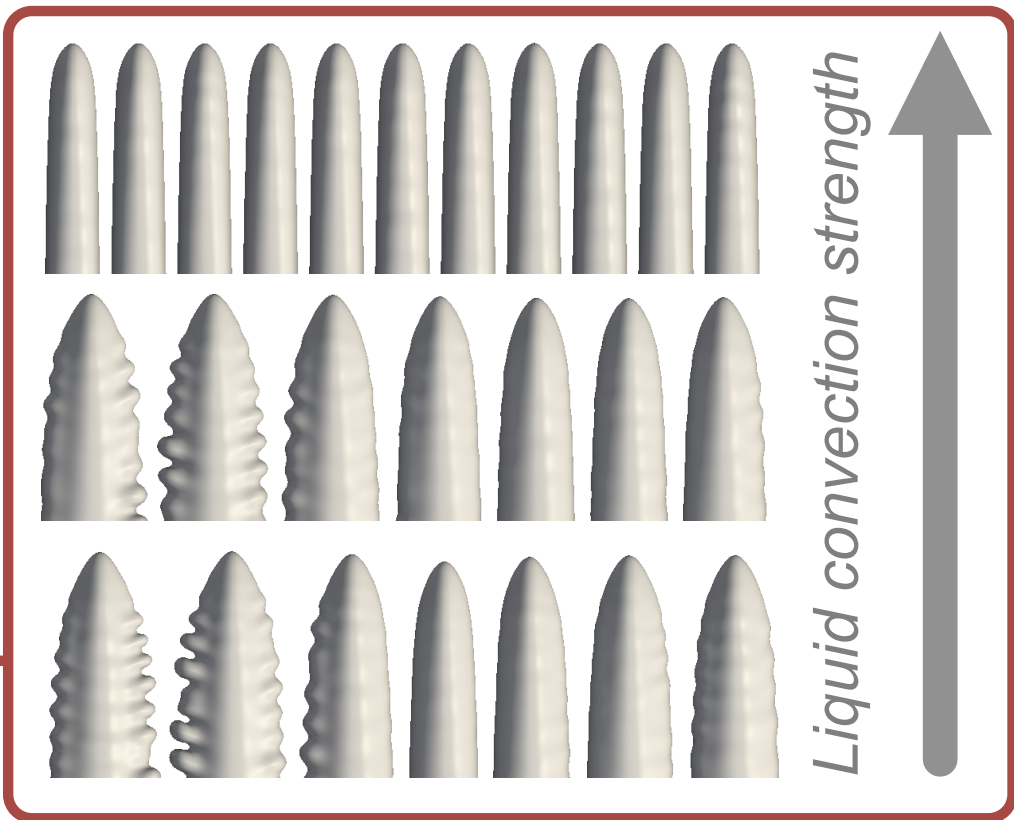

